\title{
Baxter Q-operators and representations of Yangians
}

\author{
Vladimir V. Bazhanov ${ }^{\mathrm{a}, \mathrm{b}, *}$, Rouven Frassek $^{\mathrm{c}, \mathrm{e}}$, Tomasz Łukowski ${ }^{\mathrm{c}, \mathrm{d}}$, \\ Carlo Meneghelli ${ }^{c, e}$, Matthias Staudacher ${ }^{c, e}$ \\ a Department of Theoretical Physics, Research School of Physics and Engineering, Australian National University, \\ Canberra, ACT 0200, Australia \\ ${ }^{\mathrm{b}}$ Mathematical Sciences Institute, Australian National University, Canberra, ACT 0200, Australia \\ ${ }^{\mathrm{c}}$ Institut für Mathematik und Institut für Physik, Humboldt-Universität zu Berlin, Johann von Neumann-Haus, \\ Rudower Chaussee 25, 12489 Berlin, Germany \\ d Institute of Physics, Jagellonian University, ul. Reymonta 4, 30-059 Kraków, Poland \\ e Max-Planck-Institut für Gravitationsphysik, Albert-Einstein-Institut, Am Mühlenberg 1, 14476 Potsdam, Germany
}

Received 1 March 2011; accepted 8 April 2011

Available online 15 April 2011

\begin{abstract}
We develop a new approach to Baxter Q-operators by relating them to the theory of Yangians, which are the simplest examples for quantum groups. Here we open up a new chapter in this theory and study certain degenerate solutions of the Yang-Baxter equation connected with harmonic oscillator algebras. These infinite-state solutions of the Yang-Baxter equation serve as elementary, "partonic" building blocks for other solutions via the standard fusion procedure. As a first example of the method we consider $\mathfrak{g l}(n)$ compact spin chains and derive the full hierarchy of operatorial functional equations for all related commuting transfer matrices and $\mathbf{Q}$-operators. This leads to a systematic and transparent solution of these chains, where the nested Bethe equations are derived in an entirely algebraic fashion, without any reference to the traditional Bethe Ansatz techniques.

(c) 2011 Published by Elsevier B.V.
\end{abstract}

\footnotetext{
* Corresponding author at: Mathematical Sciences Institute, Australian National University, Canberra, ACT 0200, Australia.

E-mail addresses: Vladimir.Bazhanov@anu.edu.au (V.V. Bazhanov), rfrassek@physik.hu-berlin.de (R. Frassek), lukowski@mathematik.hu-berlin.de (T. Łukowski), carlo@aei.mpg.de (C. Meneghelli), matthias@aei.mpg.de (M. Staudacher).
} 


\section{Introduction and overview}

The method of functional relations and commuting transfer matrices, introduced by Baxter in his seminal paper [1] on the exact solution of the eight-vertex model, plays a fundamental role in the theory of integrable quantum systems. It is based on an explicit algebraic construction of transfer matrices, which gives a priori knowledge about the analytic properties of their eigenvalues. A central part within this method involves the so-called $\mathbf{Q}$-operators. These operators are distinguished by the fact that zeroes of their eigenvalues precisely coincide with the roots of a certain system of algebraic equations, which arises as a part of the coordinate [2] or algebraic [3] Bethe Ansatz.

The underlying algebraic structure behind the construction of the commuting transfer matrices, termed $\mathbf{T}$-matrices or $\mathbf{T}$-operators, is by now well understood. It is connected with the simplest representations of quantum groups, which are closely related to the standard finitedimensional representations of classical Lie algebras. In contrast, the algebraic construction of the $\mathbf{Q}$-operators appears to be a more complicated and, at the same time, more interesting problem. Much progress in this direction has already been achieved from a case-by-case study of various models, see e.g. [1,2,4-11] but the problem still continues to reveal its new features.

In this paper we develop a new approach to $\mathbf{Q}$-operators by connecting them to the theory of Yangians, which are the simplest examples of quantum groups. In doing so we shall develop new aspects of the theory of infinite-dimensional representations of Yangians, naturally leading to a systematic and transparent construction of the $\mathbf{Q}$-operators. Here we illustrate our approach on the compact $\mathfrak{g l}(n)$-spin chains, ${ }^{1}$ but the results may be readily generalized to other models, and in particular to supersymmetric spin chains [12].

Let us then consider the integrable $\mathfrak{g l}(n)$-spin chain with the well-known Hamiltonian

$$
\mathbf{H}_{n}=2 \sum_{l=1}^{L}\left(1-\sum_{a, b=1}^{n} e_{a b}^{(l)} e_{b a}^{(l+1)}\right)
$$

in the presence of twisted periodic boundary conditions,

$$
e_{a b}^{(L+1)}:=e^{i\left(\Phi_{a}-\Phi_{b}\right)} e_{a b}^{(1)},
$$

where $\Phi_{1}, \Phi_{2}, \ldots, \Phi_{n}$ is a set of fixed twist parameters (or fields). Here $e_{a b}$ denotes the $n \times n$ matrix unit $\left(e_{a b}\right)_{i j}=\delta_{a i} \delta_{b j}$ and the superscript " $(l)$ " refers to the quantum space of the $l$-th spin in the chain. Each "spin" can take $n$ different values $a=1,2, \ldots, n$. It is easy to check that for the quasi-periodic boundary conditions (1.2) the numbers $m_{1}, m_{2}, \ldots, m_{n}$, counting the total number of spins of type " 1 ", " 2 ", .., " $n$ " in the chain, are conserved quantum numbers for the Hamiltonian (1.1). Due to these conservation properties this integrable model can be solved via the "nested" Bethe Ansatz technique [13,14], which leads to the well-known result for the eigenvalues of (1.1), see (6.8) below. They are expressed through solutions of the already mentioned algebraic equations, commonly called Bethe Ansatz equations.

It is relatively well known that there are different but equivalent forms of the Bethe Ansatz. In fact, it is easy to argue that there are precisely $n$ ! different Bethe Ansätze in our case, related by all possible permutations of the occupation numbers $m_{1}, m_{2}, \ldots, m_{n}$. Indeed, there are $n$ ways

\footnotetext{
1 Spin chains for which the quantum space is some finite-dimensional representation of $\mathfrak{g l}(n)$ are often referred to as $\mathfrak{s u}(n)$-spin chains to stress their compact nature. In this paper we follow the nomenclature which is customary in the Yangian literature.
} 


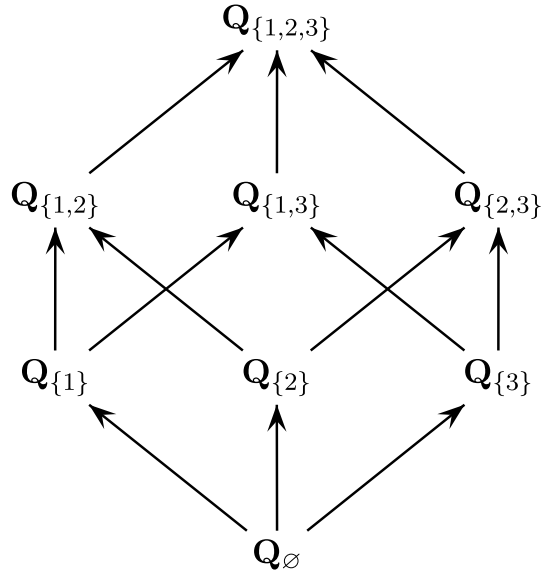

a)

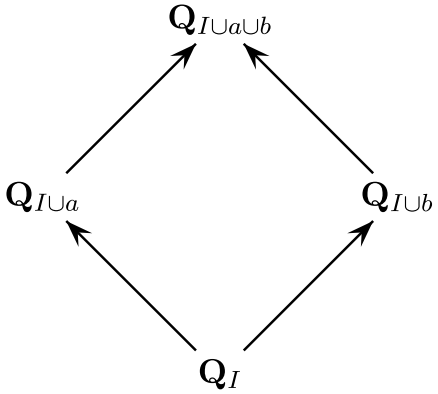

b)

Fig. 1. a) Hasse diagram for $\mathfrak{g l}(3)$; b) An elementary quadrilateral, whose bottom node corresponds to the set $I=$ $\left\{a_{1}, a_{2}, \ldots, a_{p}\right\}$.

to choose the bare vacuum state, then $n-1$ ways to proceed on the second "nested" stage of the Bethe Ansatz and so on. ${ }^{2}$ These options can be conveniently depicted by directed paths on a Hasse diagram which spans an $n$-dimensional hypercube. The nodes of the hypercube are labeled by increasing integer sets $I=\left\{a_{1}, a_{2}, \ldots, a_{p}\right\} \subseteq\{1,2, \ldots, n\}$, where $0 \leqslant p \leqslant n$. See the $n=3$ example in Fig. 1a). There are exactly $2^{n}$ nodes on the diagram and exactly $n$ ! ordered paths from the bottom to the top. Then each path is related to a particular variant of the Bethe Ansatz, while nodes on that path are related to the so-called Q-functions entering the corresponding Bethe Ansatz equations. This concise description was proposed in [16]. Note that the usual nested Bethe Ansatz proceeds from the top to the bottom of this diagram.

Our approach precisely reproduces this picture. We explicitly construct the $2^{n}$ different $\mathbf{Q}$ operators, corresponding to the nodes of the Hasse diagram, and subsequently derive the nested Bethe Ansatz equations and eigenvalues of the Hamiltonian (1.1) without any reference to the eigenvector construction. The different $\mathbf{Q}$-operators are not functionally independent. They satisfy the so-called Hirota equations, defined on the direct product of the $n$-dimensional hypercube and the real line. The equations have the same form for every quadrilateral of the Hasse diagram (see Fig. 1b)),

$$
\Delta_{\{a, b\}} \mathbf{Q}_{I \cup a \cup b}(z) \mathbf{Q}_{I}(z)=\mathbf{Q}_{I \cup a}\left(z-\frac{1}{2}\right) \mathbf{Q}_{I \cup b}\left(z+\frac{1}{2}\right)-\mathbf{Q}_{I \cup b}\left(z-\frac{1}{2}\right) \mathbf{Q}_{I \cup a}\left(z+\frac{1}{2}\right),
$$

where

$$
\Delta_{\{a, b\}}=2 i \sin \left(\frac{\Phi_{a}-\Phi_{b}}{2}\right)
$$

\footnotetext{
2 One should keep in mind, however, that the above argument only applies to the case where all fields $\Phi_{a}$ take generic, non-zero values. If some or all of the fields vanish, or else take certain special values, only a few of the Bethe Ansätze are well defined, while other ones typically suffer from multiple roots or the so-called "beyond the equator" problem [15].
} 
and the expression $I \cup a$ denote the union of the sets $I$ and the one-element set $\{a\}$. The reader might be aware that the Hirota equations frequently arise in the analysis of quantum integrable models, see e.g. [17]. Eq. (1.3) for the Q-operators and their eigenvalues were gradually developed for various models related to $\mathfrak{g l}(n)$ algebra in $[6,18-20]$. The form of the operatorial equations (1.3) presented here exactly coincide with that of the eigenvalue equations of Ref. [16]. ${ }^{3}$

The most essential algebraic property of the $\mathbf{Q}$-operators is concisely expressed by the single factorization relation

$$
\mathbf{T}^{+}\left(z, \Lambda_{n}\right) \simeq \mathbf{Q}_{\{1\}}\left(z+\lambda_{1}^{\prime}\right) \mathbf{Q}_{\{2\}}\left(z+\lambda_{2}^{\prime}\right) \cdots \mathbf{Q}_{\{n\}}\left(z+\lambda_{n}^{\prime}\right)
$$

where $\mathbf{T}^{+}\left(z, \Lambda_{n}\right)$ is the transfer matrix associated with a highest weight infinite-dimensional representation (Verma module) with the $\mathfrak{g l}(n)$-weights $\Lambda_{n}=\left(\lambda_{1}, \lambda_{2}, \ldots, \lambda_{n}\right)$. Here $\Lambda_{n}^{\prime}=\Lambda_{n}+\rho_{n}$ denotes the weights shifted by the half sum of the positive roots, $\rho_{n}$ of the algebra $\mathfrak{s l}(n)$. Remarkably, this relation alone allows to derive all functional relations, satisfied by various "fusion" transfer matrices and $\mathbf{Q}$-operators. As a result the model may then be solved in an entirely algebraic fashion. In particular, the transfer matrices

$$
\mathbf{T}\left(z, \Lambda_{n}\right) \simeq \operatorname{det}\left\|\mathbf{Q}_{i}\left(z+\lambda_{j}^{\prime}\right)\right\|_{1<i, j<n}
$$

associated with finite-dimensional representations, are expressed as the determinant of a matrix constructed from Q-operators. This approach was originally developed mainly for field theory models connected with quantized (or $q$-deformed) affine algebras $[6,22,19,23,24]$.

In [11] we applied the same idea to solve the famous Heisenberg XXX spin chain corresponding to $n=2$ in (1.1), which is much simpler than its $q$-deformed counterparts. This allowed us to observe some new algebraic structures which were obscured or did not manifest themselves for field theory models. We found that for the XXX model the relation (1.5) arises from very elegant factorization properties of the $\mathfrak{g l}(2)$-invariant Lax operator

$$
\mathcal{L}^{+}\left(z, \Lambda_{2}\right)=z+\sum_{a=1}^{3} \sigma_{a} \otimes \mathbf{J}^{a}=\left(\begin{array}{cc}
z+\mathbf{J}^{3} & \mathbf{J}^{-} \\
\mathbf{J}^{+} & z-\mathbf{J}^{3}
\end{array}\right),
$$

which acts on the tensor product of a spin- $\frac{1}{2}$ module $\mathbb{C}^{2}$ and the infinite-dimensional highest weight representation of $\mathfrak{g l}(2)$ with an arbitrary spin $j$. The $\mathfrak{g l}(2)$ generators $\mathbf{J}^{3}, \mathbf{J}^{ \pm}$in this case are realized in the Holstein-Primakoff form,

$$
\mathbf{J}^{-}=\mathbf{b}^{\dagger}\left(\mathbf{b}^{\dagger} \mathbf{b}-2 j\right), \quad \mathbf{J}^{+}=-\mathbf{b}, \quad \mathbf{J}^{3}=j-\mathbf{b}^{\dagger} \mathbf{b},
$$

where $\mathbf{b}^{\dagger}$ and $\mathbf{b}$ are generators of the oscillator algebra

$$
\mathcal{H}: \quad\left[\mathbf{b}, \mathbf{b}^{\dagger}\right]=1, \quad \mathbf{h}=\mathbf{b}^{\dagger} \mathbf{b}+\frac{1}{2} .
$$

Here we use $\mathfrak{g l}(2)$ representation labels, such that $\Lambda_{2}=(j,-j)$. The factorization in question involves the two simpler "constituent" $\mathbf{L}$-operators

$$
\mathbf{L}_{1}(z)=\left(\begin{array}{cc}
z-\mathbf{h}_{1} & \mathbf{b}_{1}^{\dagger} \\
-\mathbf{b}_{1} & 1
\end{array}\right) \quad \text { and } \quad \mathbf{L}_{2}(z)=\left(\begin{array}{cc}
1 & -\mathbf{b}_{2} \\
\mathbf{b}_{2}^{\dagger} & z-\mathbf{h}_{2}
\end{array}\right),
$$

with two sets of mutually commuting oscillators. The precise factorization formula reads

\footnotetext{
3 There are also various supersymmetric extensions of (1.3), the related bibliography can be found in [16,21].
} 


$$
\left(\begin{array}{cc}
z_{1}-\mathbf{h}_{1} & \mathbf{b}_{1}^{\dagger} \\
-\mathbf{b}_{1} & 1
\end{array}\right)\left(\begin{array}{cc}
1 & -\mathbf{b}_{2} \\
\mathbf{b}_{2}^{\dagger} & z_{2}-\mathbf{h}_{2}
\end{array}\right)=e^{\mathbf{b}_{1}^{+} \mathbf{b}_{2}^{+}}\left(\begin{array}{cc}
z+\mathbf{J}^{3} & \mathbf{J}^{-} \\
\mathbf{J}^{+} & z-\mathbf{J}^{3}
\end{array}\right)\left(\begin{array}{cc}
1 & -\mathbf{b}_{2} \\
0 & 1
\end{array}\right) e^{-\mathbf{b}_{1}^{+} \mathbf{b}_{2}^{+}},
$$

where

$$
z_{1}=z+j+\frac{1}{2}, \quad z_{2}=z-j-\frac{1}{2},
$$

and the generators $\mathbf{J}^{3}, \mathbf{J}^{ \pm}$, are realized in the Holstein-Primakoff form (1.8) with $\mathbf{b}^{\dagger}$ and $\mathbf{b}$ replaced by $\mathbf{b}_{1}^{\dagger}$ and $\mathbf{b}_{1}$. It is useful to rewrite (1.11) in a compact form

$$
\mathbf{L}_{1}\left(z_{1}\right) \mathbf{L}_{2}\left(z_{2}\right)=\mathcal{S} \mathcal{L}^{+}\left(z, \Lambda_{2}\right) \mathbb{G} \mathcal{S}^{-1},
$$

where

$$
\mathcal{S}=e^{\mathbf{b}_{1}^{+} \mathbf{b}_{2}^{+}}, \quad \mathbb{G}=\left(\begin{array}{cc}
1 & -\mathbf{b}_{2} \\
0 & 1
\end{array}\right) .
$$

A similar formula for the reversed order product $\mathbf{L}_{2} \mathbf{L}_{1}$ can also be found in [11]. These factorization equations were used to derive the $n=2$ version of (1.5) as well as the complete hierarchy of all functional relations, leading to a new algebraic solution of the XXX magnetic spin chain.

It is interesting to note that the LHS of (1.11) contains two operators (1.10), which are first order polynomials in the spectral parameter $z$, and, at the same time, their product in the RHS is also a first order polynomial in $z$. This is explained by the fact that in (1.10) terms linear in $z$ are proportional to two different degenerate matrices whose product is equal to zero. Thus, in contrast to (1.7), where the term containing $z$ is proportional to the unit matrix, the operators (1.10) start from degenerate matrices.

By extending this key observation to the $\mathfrak{g l}(n)$ case, we have completely classified all first order $\mathbf{L}$-operators and studied their fusion and factorization properties. In general they correspond to some special infinite-dimensional representations of the Yangian $Y(\mathfrak{g l}(n))$ related to a direct product of $\mathfrak{g l}(p) \otimes \mathcal{H}^{p(n-p)}$, involving the algebra $\mathfrak{g l}(p)$ with $p \leqslant n$, and a number oscillator algebras $\mathcal{H}$. In particular, we find that the $\mathfrak{g l}(n)$ Lax operator

$$
\mathcal{L}^{+}\left(z, \Lambda_{n}\right)=z+\sum_{a, b} e_{a b} \otimes \mathbf{J}_{b a}
$$

for infinite-dimensional highest weight representations of the $\mathfrak{g l}(n)$ generators $\mathbf{J}_{a b}$ factorizes into $n$ partonic Lax operators $\mathbf{L}_{a}(z)(a=1, \ldots, n)$,

$$
\mathbf{L}_{a}(z)=\left(\begin{array}{cccccccc}
1 & \multicolumn{7}{c}{-\mathbf{b}_{1, a}} \\
& \ddots & \multicolumn{5}{c}{} & \vdots \\
& & 1 & -\mathbf{b}_{a-1, a} & & & \\
\mathbf{b}_{a, 1}^{\dagger} & \cdots & \mathbf{b}_{a, a-1}^{\dagger} & z-\mathbf{h}_{a} & \mathbf{b}_{a, a+1}^{\dagger} & \cdots & \mathbf{b}_{a, n}^{\dagger} \\
& & & -\mathbf{b}_{a+1, a} & 1 & & \\
& & & \vdots & & \ddots & \\
& & & -\mathbf{b}_{n, a} & & & 1
\end{array}\right),
$$

which is the generalization of (1.10) for the $\mathfrak{g l}(n)$ case. Each of these operators contains $n-1$ independent oscillator pairs $\left(\mathbf{b}_{b a}, \mathbf{b}_{a b}^{\dagger}\right), b=1,2, \ldots, n, b \neq a$,

$$
\left[\mathbf{b}_{b a}, \mathbf{b}_{a b}^{\dagger}\right]=1, \quad \mathbf{h}_{a}=\sum_{b \neq a}\left(\mathbf{b}_{a b}^{\dagger} \mathbf{b}_{b a}+\frac{1}{2}\right) .
$$


The factorization formula, generalizing (1.13), now reads

$$
\mathbf{L}_{1}\left(z+\lambda_{1}^{\prime}\right) \mathbf{L}_{2}\left(z+\lambda_{2}^{\prime}\right) \cdots \mathbf{L}_{n}\left(z+\lambda_{n}^{\prime}\right)=\mathcal{S}_{\mathcal{L}} \mathcal{L}^{+}\left(z \mid \Lambda_{n}\right) \mathbb{G}_{\mathcal{L}} \mathcal{S}_{\mathcal{L}}^{-1}
$$

Here the shifted weights $\Lambda_{n}^{\prime}=\Lambda_{n}+\rho_{n}$ are as in (1.5), $\rho_{n}$ is given by (4.18) and the quantities $\mathcal{S}_{\mathcal{L}}$ and $\mathbb{G}_{\mathcal{L}}$ are generalizations of those in (1.14). Eq. (1.18) immediately implies the factorization relation $(1.5)$.

Finally, note that the formula (1.16) (and its generalization (2.20)) define extremely simple first order solutions of the Yang-Baxter equation. The solutions are new and were not previously considered in the literature. We will now proceed to derive and precise all above statements.

\section{The Yang-Baxter equation and representations of Yangians}

The Hamiltonian (1.1) with twisted boundary conditions commutes with a large commuting family of $\mathbf{T}$ - and $\mathbf{Q}$-operators. In this paper we explicitly construct these operators via traces of certain monodromy matrices associated with infinite-dimensional representations of the harmonic oscillator algebra. To do this we need to find appropriate solutions of the Yang-Baxter equation

$$
\mathbf{R}\left(z_{1}-z_{2}\right)\left(\mathbb{L}\left(z_{1}\right) \otimes 1\right)\left(1 \otimes \mathbb{L}\left(z_{2}\right)\right)=\left(1 \otimes \mathbb{L}\left(z_{2}\right)\right)\left(\mathbb{L}\left(z_{1}\right) \otimes 1\right) \mathbf{R}\left(z_{1}-z_{2}\right),
$$

where $\mathbf{R}(z)$ is an $n^{2} \times n^{2}$ matrix,

$$
\mathbf{R}(z): \quad \mathbb{C}^{n} \otimes \mathbb{C}^{n} \rightarrow \mathbb{C}^{n} \otimes \mathbb{C}^{n}, \quad \mathbf{R}(z)=z+\mathbf{P},
$$

acting in the direct product of two $n$-dimensional spaces $\mathbb{C}^{n} \otimes \mathbb{C}^{n}$. The operator $\mathbf{P}$ permutes the factors in this product. The operator $\mathbb{L}(z)$ is an $n \times n$ matrix, acting in a single copy of the space $\mathbb{C}^{n}$, whose matrix elements are operator-valued functions of the variable $z$ belonging to some associative algebra $\mathcal{Y}$. To be more precise, the Yang-Baxter equation (2.1) provides defining relations of the Yangian algebra $\mathcal{Y}=Y(\mathfrak{g l}(n))$, introduced by Drinfeld [25] (for a recent comprehensive review see [26]).

Let $L_{i j}(z), i, j=1,2, \ldots, n$, denote the matrix elements of $\mathbb{L}(z)$. From (2.1) it follows that

$$
(y-x)\left[L_{i j}(x), L_{k \ell}(y)\right]=L_{k j}(x) L_{i \ell}(y)-L_{k j}(y) L_{i \ell}(x), \quad i, j, k, \ell=1,2, \ldots, n .
$$

Writing $L_{i j}(z)$ as a Laurent series with operator-valued coefficients

$$
L_{i j}(z)=L_{i j}^{(0)}+L_{i j}^{(1)} z^{-1}+L_{i j}^{(2)} z^{-2}+\cdots,
$$

one obtains an infinite set of commutation relations

$$
\left[L_{i j}^{(r)}, L_{k \ell}^{(s)}\right]=\sum_{a=1}^{\min (r, s)}\left(L_{k j}^{(r+s-a)} L_{i \ell}^{(a-1)}-L_{k j}^{(a-1)} L_{i \ell}^{(r+s-a)}\right)
$$

for the elements $L_{i j}^{(r)}, r=0,1,2, \ldots, \infty$. Thus the problem of solving Eq. (2.1) reduces to the construction of representations of the infinite-dimensional quadratic algebra (2.5). In our approach the most important role is played by the simplest representations, where the series (2.4) truncates after the second term. To within a trivial change in the normalization these representations correspond to $\mathbf{L}$-operators, which are first order polynomials in the parameter $z$. All previously known L-operators of this type can be brought to the form

$$
\mathcal{L}_{i j}(z)=z \delta_{i j}+\mathcal{J}_{j i},
$$


where $\mathcal{J}_{i j}, i, j=1,2, \ldots, n$ denotes the standard set of generating elements of the algebra $\mathfrak{g l}(n)$,

$$
\left[\mathcal{J}_{i j}, \mathcal{J}_{k \ell}\right]=\delta_{k j} \mathcal{J}_{i \ell}-\delta_{i \ell} \mathcal{J}_{k j}
$$

The Yang-Baxter equation for the $\mathbf{L}$-operator (2.6) is satisfied on the algebraic level by virtue of the commutation relations (2.7). Therefore one can choose in (2.6) arbitrary $\mathfrak{g l}(n)$ representations for the generators $\mathcal{J}_{i j}$. It is obvious that the addition of a constant to the spectral parameter $z$ in (2.6) can be compensated by the subtraction of the same constant from the central element

$$
\mathcal{C}_{n}=\mathcal{J}_{11}+\mathcal{J}_{22}+\cdots+\mathcal{J}_{n n}
$$

of the algebra (2.7). Therefore it is tempting to eliminate this spurious degree of freedom by, for instance, imposing the condition $\mathcal{C}_{n}=0$ and restricting (2.7) to the algebra $\mathfrak{s l}(n)$. Here we will not do so, but will instead work with the full algebra $\mathfrak{g l}(n)$. This is helpful for clearly exposing the Weyl group symmetry of the problem at hand.

We will now show that, excitingly, there exist further first order L-operators, different from (2.6). We will present their complete classification. To begin, let us recall a simple symmetry of the Yang-Baxter equation (2.1). The $R$-matrix (2.2) is $G L(n)$-invariant in the sense

$$
\mathbf{R}(z)=(\mathbb{G} \otimes \mathbb{G}) \mathbf{R}(z)(\mathbb{G} \otimes \mathbb{G})^{-1}, \quad \mathbb{G} \in G L(n),
$$

where $\mathbb{G}$ is any non-degenerate $n \times n$ matrix. It follows that if $\mathbb{L}(z)$ satisfies $(2.1)$, then any other operator of the form

$$
\tilde{\mathbb{L}}(z)=\mathbb{F} \mathbb{L}(z) \mathbb{G}, \quad \mathbb{F}, \mathbb{G} \in G L(n),
$$

with arbitrary $\mathbb{F}, \mathbb{G} \in G L(n)$ satisfies again the same equation. Furthermore, the matrices $\mathbb{F}, \mathbb{G}$ may contain operator-valued matrix elements, as long as these commute among themselves and with all other elements of $\mathbb{L}(z)$.

From (2.5) it then immediately follows that the elements $L_{i j}^{(0)}$ are central, i.e. they commute among themselves and with all $L_{i j}^{(r)}$ for $r \geqslant 1$. Therefore, we may regard $L^{(0)}$ as a numerical $n \times n$ matrix. Applying the transformations (2.10), this matrix can always be brought to diagonal form

$$
L^{(0)}=\operatorname{diag}(\underbrace{1,1, \ldots, 1}_{p \text {-times }}, \underbrace{0,0, \ldots, 0}_{(n-p) \text {-times }}), \quad p=1,2, \ldots, n,
$$

where $p$ is an integer $1 \leqslant p \leqslant n$. The number $p$ coincides with the rank of the matrix $L^{(0)}$. It is invariant under the linear transformations (2.10). Evidently, if $p=n$, the leading term in the series expansion (2.4) is the unit matrix. This case is well studied in the existing representation theory. In fact, the assumption that the series (2.4) starts with the unit matrix is usually included into the definition of the Yangian. Here we will not make this assumption, and will consider instead the more general case with arbitrary $1 \leqslant p \leqslant n$.

Let us concentrate on the simple case when the series (2.4) truncates after the second term, i.e. assume that all $L_{i j}^{(r)}=0$ for $r \geqslant 2$. It is convenient to write the only remaining non-trivial coefficient $L^{(1)}$ as a block matrix

$$
L^{(1)}=\left(\begin{array}{c:c}
A_{a b} & B_{a \dot{b}} \\
\hdashline C_{\dot{a} b} & D_{\dot{a} \dot{b}}
\end{array}\right)
$$


where $A, B, C$ and $D$ are operator-valued matrices of dimensions $p \times p, p \times(n-p),(n-p) \times p$ and $(n-p) \times(n-p)$, respectively. We furthermore assume that all undotted indices run over the values $\{1,2, \ldots, p\}$, whereas their dotted counterparts take on the values $\{p+1, \ldots, n\}$.

$$
1 \leqslant a, b \leqslant p, \quad p+1 \leqslant \dot{a}, \dot{b} \leqslant n .
$$

Substituting (2.11) and (2.12) into (2.5), one immediately realizes that the elements $D_{\dot{a} \dot{b}}$ are central, i.e. they commute among themselves and with all other elements of $L^{(1)}$. The other commutation relations read

$$
\begin{array}{llll}
{\left[A_{a b}, A_{c e}\right]=\delta_{a e} A_{c b}-\delta_{c b} A_{a e},} & {\left[A_{a b}, B_{c \dot{c}}\right]=-\delta_{b c} B_{a \dot{c}},} & & {\left[A_{a b}, C_{\dot{c} c}\right]=+\delta_{a c} C_{\dot{c} b},} \\
{\left[B_{a \dot{b}}, C_{\dot{a} b}\right]=\delta_{a b} D_{\dot{a} \dot{b}},} & {\left[B_{a \dot{b}}, B_{c \dot{e}}\right]=0,} & {\left[C_{\dot{a} b}, C_{\dot{c} e}\right]=0 .}
\end{array}
$$

Using the remaining freedom of making transformations (2.10), which do not affect the form of $L^{(0)}$ in (2.11), one can then bring the matrix $D$ to diagonal form with zeroes and ones on the diagonal, in similarity to (2.11). Here we are only interested in highest weight representations of the algebra (2.14). These representations admit a definition of the trace, as required for the construction of transfer matrices in Section 4 below. For this reason we only need to consider the non-degenerate case, ${ }^{4} \operatorname{det} D \neq 0$, where the diagonal form of $D$ coincides with the $(n-p) \times$ $(n-p)$ unit matrix

$$
D_{\dot{a} \dot{b}}=\delta_{\dot{a} \dot{b}}, \quad p+1 \leqslant \dot{a}, \dot{b} \leqslant n .
$$

The resulting algebra (2.14) can be realized as a direct product of the algebra $\mathfrak{g l}(p)$ with $p(n-p)$ copies of the harmonic oscillator algebra:

$$
\mathcal{A}_{n, p}=\mathfrak{g l}(p) \otimes \mathcal{H}^{\otimes p(n-p)} .
$$

Introduce $p(n-p)$ independent oscillator pairs $\left(\mathbf{b}_{\dot{a} b}, \mathbf{b}_{b \dot{a}}^{\dagger}\right)$, where $\dot{a}=p+1, \ldots, n$ and $b=$ $1, \ldots, p$, satisfying the relations

$$
\left[\mathbf{b}_{\dot{a} b}, \mathbf{b}_{c \dot{e}}^{\dagger}\right]=\delta_{\dot{a} \dot{e}} \delta_{b c} \text {. }
$$

Furthermore, let $\mathcal{J}_{a b}, a, b=1,2, \ldots, p$, denote the generators of the algebra $\mathfrak{g l}(p)$ defined by (2.7), where $n$ is replaced by $p$. The generators $\mathcal{J}_{a b}$ commute with all oscillator operators in (2.17). The connection of the algebra (2.14) with the product (2.16) is established by the following relations

$$
A_{a b}=\overline{\mathcal{J}}_{a b}-\sum_{\dot{c}=p+1}^{n}\left(\mathbf{b}_{a \dot{c}}^{\dagger} \mathbf{b}_{\dot{c} b}+\frac{1}{2} \delta_{a b}\right), \quad B_{a \dot{b}}=\mathbf{b}_{a \dot{b}}^{\dagger}, \quad C_{\dot{a} b}=-\mathbf{b}_{\dot{a} b},
$$

where the upper bar in the notation $\overline{\mathcal{J}}_{a b}$ denotes the transposition of the indices $a$ and $b$,

$$
\overline{\mathcal{J}}_{a b} \equiv \mathcal{J}_{b a} .
$$

The corresponding $\mathbf{L}$-operator can be written as a block matrix,

\footnotetext{
${ }^{4}$ It appears that for det $D=0$ the algebra (2.14) does not admit a definition for a suitable trace as needed for the construction of transfer matrices commuting with the Hamiltonian (1.1). See footnote 7 in Section 4 for additional details.
} 


$$
\mathbf{L}_{\{1,2, \ldots, p\}}(z)=\left(\begin{array}{c:c}
z \delta_{a b}+\overline{\mathcal{J}}_{a b}-\sum_{\dot{c}=p+1}^{n}\left(\mathbf{b}_{a \dot{c}}^{\dagger} \mathbf{b}_{\dot{c} b}+\frac{1}{2} \delta_{a b}\right) & \mathbf{b}_{a \dot{b}}^{\dagger} \\
\hdashline \ldots \ldots \ldots \ldots \ldots \ldots \ldots \ldots \ldots \ldots \ldots \ldots \ldots \ldots \ldots \ldots \ldots \ldots \ldots \ldots \ldots \ldots \ldots \\
-\mathbf{b}_{\dot{a} b} & \delta_{\dot{a} \dot{b}}
\end{array}\right),
$$

where the rows are labeled by the indices $a$ or $\dot{a}$ and the columns by $b$ or $\dot{b}$, in similarity to (2.12). Note that the $p \times p$ matrix of the generators of $\mathfrak{g l}(p)$, which enters the upper left block, is transposed, i.e. the $a$-th row and $b$-th column in this block contains the element $\overline{\mathcal{J}}_{a b}=\mathcal{J}_{b a}$.

The matrix (2.20) contains the parameter $z$ only in its first $p$ diagonal elements. By simultaneous permutations of rows and columns in (2.20) one can move these $z$-containing elements to $p$ arbitrary positions on the diagonal, labeled by a set of integers $I=\left\{a_{1}, a_{2}, \ldots, a_{p}\right\}$. We shall denote the $\mathbf{L}$-operator obtained in this way by $\mathbf{L}_{I}(z)$. Within this convention the operator (2.20) corresponds to the set $I=\{1,2, \ldots, p\}$, as indicated by the subscript in the LHS of this equation.

The "partonic" L-operator (1.16) is a particular case of (2.20) with $p=1$, while the standard L-operator (2.6) corresponds to $p=n$. We would like to stress that for $p<n$ the formula (2.20) yields novel solutions of the Yang-Baxter equation (2.1). The only exception is the simple case $n=2, p=1$, where this solution was previously known [27-29]. Note, also that the $n=3$ solutions can be obtained in the rational limit of trigonometric solutions obtained in [30,31].

The formula (2.20) provides an evaluation homomorphism of the infinite-dimensional Yangian algebra (2.5) into the finite-dimensional algebra (2.16),

$$
Y(\mathfrak{g l}(n)) \rightarrow \mathfrak{g l}(p) \otimes \mathcal{H}^{\otimes p(n-p)}, \quad 1 \leqslant p \leqslant n .
$$

This means that for any representation of this finite-dimensional algebra Eq. (2.20) automatically defines a representation of the Yangian and a matrix solution of the Yang-Baxter equation (2.1). Conversely, any first order matrix L-operator with a rank $p$ leading term $L^{(0)}$ and a non-degenerate matrix $D$ in (2.12) is, up to a transformation (2.10), equivalent to the canonical L-operator (2.20) with some particular representation of the algebra (2.16). It is worth noting that the transformation $(2.10)$

$$
\mathbf{L}_{\{1,2, \ldots, p\}}(z) \rightarrow \mathbb{F} \mathbf{L}_{\{1,2, \ldots, p\}}(z) \mathbb{F}^{-1},
$$

where $\mathbb{F}$ is a block diagonal matrix containing the matrices $\mathbb{F}_{p} \in G L(p), \mathbb{F}_{n-p} \in G L(n-p)$ on the diagonal, leaves the form of (2.20) unchanged.

In preparation for a necessary analysis below we need to introduce some notation for the irreducible highest weight representations of $\mathfrak{g l}(n)$. The highest weight vector $v_{0}$ is defined by the conditions

$$
\mathcal{J}_{a, a+1} v_{0}=0, \quad a=1,2, \ldots, n-1 .
$$

Here we will use $\mathfrak{g l}(n)$-type representation labels ${ }^{5} \Lambda_{n}=\left(\lambda_{1}, \lambda_{2}, \ldots, \lambda_{n}\right)$ where

$$
\mathcal{J}_{a a} v_{0}=\lambda_{a} v_{0}, \quad a=1,2, \ldots, n .
$$

\footnotetext{
5 The $\mathfrak{s l}(n)$-type weights $\mu=\left(\mu_{1}, \mu_{2}, \ldots, \mu_{n-1}\right)$,

$$
\mu_{a}=\lambda_{a}-\lambda_{a+1}, \quad a=1,2, \ldots, n-1,
$$
}


We will denote by $\pi_{\Lambda_{n}}^{+}$the corresponding infinite-dimensional highest weight representation with arbitrary weights, and by $\pi_{\Lambda_{n}}$ the finite-dimensional irreducible representation with

$$
\lambda_{1} \geqslant \lambda_{2} \geqslant \cdots \geqslant \lambda_{n}, \quad \lambda_{a}-\lambda_{b} \in \mathbb{Z} .
$$

The analysis of this section extends the previous results of [27] devoted to $n=2$ case. The properties of the finite-dimensional representation of the Yangian $Y(\mathfrak{g l}(2))$ associated with the L-operator (2.6) can be found in $[32,33,26]$.

\section{Fusion and factorization of L-operators}

An essential part of our analysis in the following is based on some remarkable decomposition properties of the product of two L-operators of the form (2.20). The Yangian $\mathcal{Y}=Y(\mathfrak{g l}(n))$ is a Hopf algebra, see e.g. [26]. In particular, its co-multiplication

$$
\mathcal{Y} \rightarrow \mathcal{Y} \otimes \mathcal{Y}
$$

is generated by the matrix product of two $\mathbf{L}$-operators, corresponding to two different copies of $\mathcal{Y}$ appearing on the RHS of (3.1).

Our main observation is related to the co-product of two operators $\mathbf{L}_{I}(z)$ and $\mathbf{L}_{J}(z)$, defined by (2.20) for two non-intersecting sets $I \cap J=\varnothing$,

$$
\mathbf{L}(z)=\mathbf{L}_{I}^{[1]}\left(z+z_{1}\right) \mathbf{L}_{J}^{[2]}\left(z+z_{2}\right),
$$

where the quantities $z_{1,2}$ denote arbitrary constants, and the notation [1] and [2] corresponds to, respectively, the left and right copies of $\mathcal{Y}$ on the RHS of (3.1). Let the sets $I$ and $J$ contain $p_{1}$ and $p_{2}$ elements, respectively. It is obvious that

(i) the product (3.2) is of the first order in the variable $z$, and that

(ii) the matrix rank of the term linear in $z$ in (3.2) is equal to $p_{1}+p_{2}$.

The simple meaning of the co-multiplication is that the matrix product of two $\mathbf{L}$-operators, each of which satisfies by itself the Yang-Baxter equation (2.1), solves this equation as well. All solutions which possess the above properties (i) and (ii) were classified in the previous section. Therefore, by using a transformation of type (2.9), the RHS of (3.2) can be brought to a particular case of the canonical form (2.20) with $p=p_{1}+p_{2}$. It turns out, however, that the expressions for the matrix elements of the resulting $\mathbf{L}$-operator are rather complicated and their explicit connection to those of (2.20) is far from obvious, even though these elements satisfy the same commutation relations. In order to make this connection more transparent we apply a suitable operatorial similarity transformation $\mathcal{S}$ to each matrix element such that it rearranges the basis of the oscillator algebras contained in $\mathcal{A}_{n, p_{1}} \otimes \mathcal{A}_{n, p_{2}}$. Furthermore, the formula (3.2) contains two constants $z_{1}$ and $z_{2}$. Obviously only their difference is an essential parameter, whereas the sum may be absorbed into the spectral parameter $z$. Therefore, without loss of generality, one can set

$$
z_{1}=\lambda+\frac{p_{2}}{2}, \quad z_{2}=-\frac{p_{1}}{2},
$$

where $\lambda$ is arbitrary. This particular parametrization is chosen to simplify the subsequent formulae. 
Proceeding as described above, one obtains

$$
\mathbf{L}(z)=\mathbf{L}_{I}^{[1]}\left(z+\lambda+\frac{p_{2}}{2}\right) \mathbf{L}_{J}^{[2]}\left(z-\frac{p_{1}}{2}\right)=\mathcal{S}\left(\mathbf{L}_{I \cup J}(z) \mathbb{G}\right) \mathcal{S}^{-1},
$$

where $\mathbb{G}$ is a $z$-independent matrix, whose elements commute among themselves and with all elements of $\mathbf{L}_{I \cup J}(z)$. It should be stressed that the resulting $\mathbf{L}$-operator $\mathbf{L}_{I \cup J}(z)$ is only a special case of (2.20), since it is connected to some specific realization of the algebra $\mathcal{A}_{n, p_{1}+p_{2}}$ in terms of the direct product of the two algebras $\mathcal{A}_{n, p_{1}} \otimes \mathcal{A}_{n, p_{2}}$ as defined in (2.16). Note that the RHS of (3.4) is of course a particular case of the transformation (2.10) with $\mathbb{F} \equiv 1$. The explicit expression for the matrices appearing in (3.4) are presented below. Some additional details of calculations are given in Appendix A.

By permuting rows and columns any two non-intersecting sets $I$ and $J$ can be reduced to the case when $I=\left\{1, \ldots, p_{1}\right\}$ and $J=\left\{p_{1}+1, \ldots, p_{1}+p_{2}\right\}$. So it is sufficient to consider this case only. Introduce three types of indices

$$
a, b, \in I, \quad \dot{a}, \dot{b} \in J, \quad \ddot{a}, \ddot{b} \in\left\{p_{1}+p_{2}+1, \ldots, n\right\} .
$$

It will be convenient to rewrite $(2.20)$ as a $3 \times 3$ block matrix

$$
\begin{aligned}
& \mathbf{L}_{I}^{[1]}(z)=\mathbf{L}_{\left\{1,2, \ldots, p_{1}\right\}}^{[1]}(z)
\end{aligned}
$$

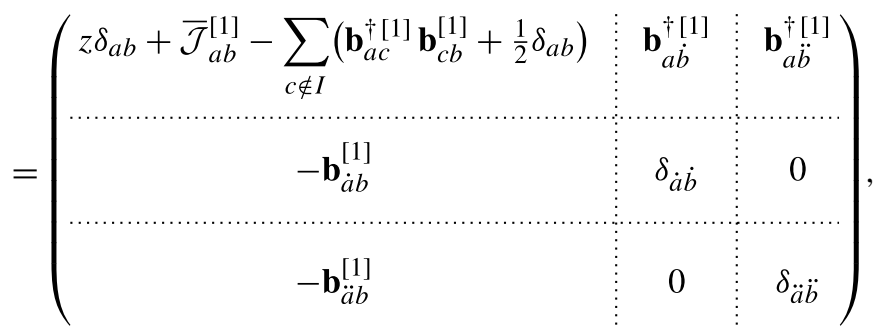

where the size of the diagonal blocks is equal to $p_{1} \times p_{1}, p_{2} \times p_{2}$ and $\left(n-p_{1}-p_{2}\right) \times(n-$ $\left.p_{1}-p_{2}\right)$, respectively. The superscript "[1]" indicates that the corresponding operators belong to the "first" algebra in the co-multiplication (3.1), which in the considered case is realized by the algebra $\mathcal{A}_{n, p_{1}}$ defined in (2.16). Similarly, one can write $\mathbf{L}_{J}^{[2]}(z)$ as

$$
\begin{aligned}
& \mathbf{L}_{J}^{[2]}(z)=\mathbf{L}_{\left\{p_{1}+1, \ldots, p_{1}+p_{2}\right\}}^{[2]}(z)
\end{aligned}
$$

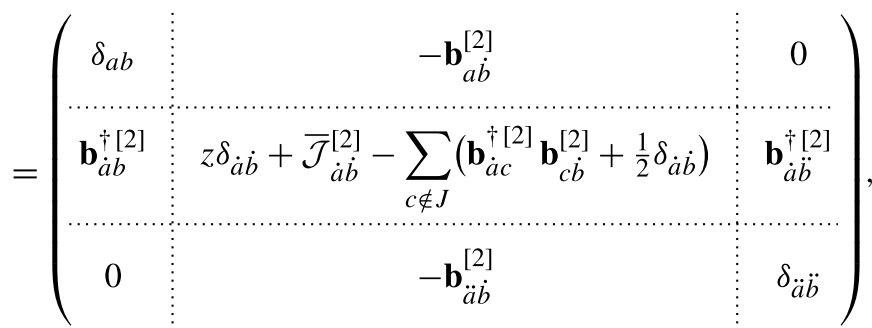

where superscript "[2]" labels operators from the "second" algebra, which is the algebra $\mathcal{A}_{n, p_{2}}$. By construction, all operators labeled by the superscript "[1]" commute with those labeled by the superscript "[2]". Recall also that the algebra (2.16) has a direct product structure, so the generators $\mathcal{J}_{a b}^{[1]}$ and $\mathcal{J}_{\dot{a} \dot{b}}^{[2]}$ commute with all oscillator operators.

With the notation introduced above the similarity transformation $\mathcal{S}$ has the form

$$
\mathcal{S}=\mathcal{S}_{1} \mathcal{S}_{2}
$$


where

$$
\mathcal{S}_{1}=\exp \left(\sum_{c \in I} \sum_{\dot{c} \in J} \mathbf{b}_{c \dot{c}}^{\dagger[1]} \mathbf{b}_{\dot{c} c}^{\dagger[2]}\right),
$$

and

$$
\mathcal{S}_{2}=\exp \left(\sum_{c \in I} \sum_{\dot{c} \in J} \sum_{\ddot{c} \notin I \cup J} \mathbf{b}_{c \dot{c}}^{\dagger[1]} \mathbf{b}_{\dot{c} \ddot{c}}^{\dagger[2]} \mathbf{b}_{\ddot{c} c}^{[1]}\right) .
$$

The matrix $\mathbb{G}$ has the form

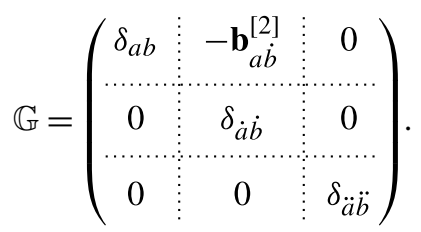

The similarity transform $\mathcal{S}_{1}$ makes it manifest that the matrix entries of $\mathbf{L}_{I \cup J}(z)$ commute with the entries of $\mathbb{G}$, see also (3.17). The similarity transform $\mathcal{S}_{2}$ brings $\mathbf{L}_{I \cup J}(z)$ to the form (2.20).

Finally, we want to write the operator $\mathbf{L}_{I \cup J}(z)$ in (3.4) in the form (2.20) with $p=p_{1}+p_{2}$. To do this we need to make the following identifications for the generators $\mathcal{J}_{i j}, i, j=1, \ldots, p_{1}+p_{2}$, in the upper diagonal block of (2.20)

$$
\begin{aligned}
& \overline{\mathbf{J}}_{a b}=\overline{\mathcal{J}}_{a b}^{[1]}-\sum_{\dot{c} \in J} \mathbf{b}_{a \dot{c}}^{\dagger[1]} \mathbf{b}_{\dot{c} b}^{[1]}+\lambda \delta_{a b}, \\
& \overline{\mathbf{J}}_{\dot{a} \dot{b}}=\overline{\mathcal{J}}_{\dot{a} \dot{b}}^{[2]}+\sum_{c \in I} \mathbf{b}_{c \dot{a}}^{\dagger[1]} \mathbf{b}_{\dot{b} c}^{[1]}, \\
& \overline{\mathbf{J}}_{\dot{a} b}=-\mathbf{b}_{\dot{a} b}^{[1]}, \\
& \overline{\mathbf{J}}_{a \dot{b}}=\left(\sum_{c \in I} \sum_{\dot{c} \in J} \mathbf{b}_{a \dot{c}}^{\dagger[1]} \mathbf{b}_{c \dot{b}}^{\dagger[1]} \mathbf{b}_{\dot{c} c}^{[1]}\right)-\lambda \mathbf{b}_{a \dot{b}}^{\dagger[1]}+\sum_{\dot{c} \in J} \mathbf{b}_{a \dot{c}}^{\dagger[1]} \overline{\mathcal{J}}_{\dot{c} \dot{b}}^{[2]}-\sum_{c \in I} \overline{\mathcal{J}}_{a c}^{[1]} \mathbf{b}_{c \dot{b}}^{\dagger[1]},
\end{aligned}
$$

where we have used the convention (3.5) for numerating indices and $\overline{\mathbf{J}}_{i j} \equiv \mathbf{J}_{j i}$.

Furthermore, let the indices A, B run over the values $1,2, \ldots, p_{1}+p_{2}$ and $\dot{\mathrm{A}}, \dot{\mathrm{B}}$ over the values $p_{1}+p_{2}+1, \ldots, n$. Introduce operators

$$
\mathbf{c}_{\dot{\mathrm{A}} \mathrm{B}}=\left\{\begin{array}{ll}
\mathbf{b}_{\dot{\mathrm{A}} \mathrm{B}}^{[1]}, & \mathrm{B} \in I, \\
\mathbf{b}_{\dot{\mathrm{A}} \mathrm{B}}^{[2]}, & \mathrm{B} \in J,
\end{array} \quad \mathbf{c}_{\mathrm{A} \dot{\mathrm{B}}}^{\dagger}= \begin{cases}\mathbf{b}_{\mathrm{A} \dot{\mathrm{B}}}^{\dagger[1]}, & \mathrm{A} \in I, \\
\mathbf{b}_{\mathrm{A} \dot{\mathrm{B}}}^{\dagger[2]}, & \mathrm{A} \in J .\end{cases}\right.
$$

Then the $\mathbf{L}$-operator $\mathbf{L}_{I \cup J}(z)$ from (3.4) can be written as

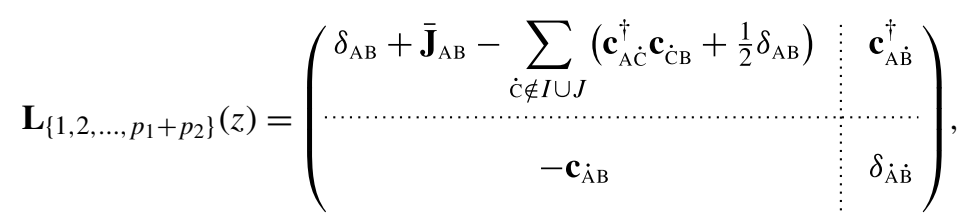

which has the required form as in (2.20).

The formulae (3.12) give a homomorphism of the algebra $\mathfrak{g l}\left(p_{1}+p_{2}\right)$ into the direct product

$$
\mathfrak{g l}\left(p_{1}+p_{2}\right) \rightarrow \mathfrak{g l}\left(p_{1}\right) \otimes \mathfrak{g l}\left(p_{2}\right) \otimes \mathcal{H}^{\otimes p_{1} p_{2}}=\mathcal{B}_{p_{1}, p_{2}},
$$


which for $p_{1,2} \neq 0$ has only infinite-dimensional representations ${ }^{6}$ (similar representations appeared in [34]). An important feature of this map is that if one choses highest weight representations for both algebras $\mathfrak{g l}\left(p_{1}\right)$ and $\mathfrak{g l}\left(p_{2}\right)$ then the formulae (3.12) define a highest weight representation of $\mathfrak{g l}\left(p_{1}+p_{2}\right)$. It is easy to check that the conditions (2.23) are satisfied on the product of the corresponding highest weight vectors $v_{0}^{[1]}, v_{0}^{[2]}$ and the standard Fock vacuum for all oscillator algebras appearing in (3.12). The $\mathfrak{g l}\left(p_{1}+p_{2}\right)$-weight of the resulting representation is easy to obtain from (3.12)

$$
\Lambda_{p_{1}+p_{2}}=\left(\lambda_{1}^{[1]}+\lambda, \lambda_{2}^{[1]}+\lambda, \ldots, \lambda_{p_{1}}^{[1]}+\lambda, \lambda_{1}^{[2]}, \lambda_{2}^{[2]}, \ldots, \lambda_{p_{2}}^{[2]}\right),
$$

where $\lambda$ is an arbitrary parameter from (3.4).

The $\mathbf{L}$-operators in the first product in (3.4) have the superscripts [1] and [2], which indicate that they belong to different algebras (2.16) with $p=p_{1}$ and $p=p_{2}$, respectively. By the same reason it is useful to rewrite the RHS of (3.4) supplying similar superscripts

$$
(3.4)=\mathcal{S}\left(\mathbf{L}_{I \cup J}^{\left[1^{\prime}\right]}(z) \mathbb{G}^{\left[2^{\prime}\right]}\right) \mathcal{S}^{-1},
$$

where the superscript $\left[1^{\prime}\right]$ indicates the algebra (3.15) and the superscript [2'] indicates the product of oscillator algebras $\mathcal{H}^{\otimes p_{1} p_{2}}$. Note that the matrix $\mathbb{G}$ could be considered as a $z$-independent L-operator, also satisfying the Yang-Baxter equation (2.1). In view of this Eq. (3.17) also describes the co-multiplication of two representations of the Yangian.

Consider now some particular consequences of formula (3.4). Using it iteratively with $p_{1}=1$ and taking into account (3.16) one can obtain an arbitrary product of the elementary $\mathbf{L}$-operators (1.16). Let $I=\left(a_{1}, \ldots, a_{p}\right)$ be an ordered integer set, $1 \leqslant a_{1}<\cdots<a_{p} \leqslant n$, and $\mathcal{L}_{I}^{+}\left(z \mid \Lambda_{p}\right)$ a specialization of the $\mathbf{L}$-operator (2.20) to the infinite-dimensional highest weight representation $\pi_{\Lambda_{p}}^{+}$of the algebra $\mathfrak{g l}(p)$

$$
\mathbf{L}_{I}^{+}\left(z \mid \Lambda_{p}\right)=\pi_{\Lambda_{p}}^{+}\left[\mathbf{L}_{I}(z)\right], \quad \Lambda_{p}=\left(\lambda_{1}, \lambda_{2}, \ldots, \lambda_{p}\right) .
$$

Define also the shifted weights (cf. (4.18))

$$
\Lambda_{p}^{\prime}=\left(\lambda_{1}^{\prime}, \lambda_{2}^{\prime}, \ldots, \lambda_{p}^{\prime}\right), \quad \lambda_{j}^{\prime}=\lambda_{j}+\frac{p-2 j+1}{2}, \quad j=1, \ldots, p .
$$

Then it follows that from (3.4)

$$
\mathbf{L}_{a_{1}}\left(z+\lambda_{1}^{\prime}\right) \mathbf{L}_{a_{2}}\left(z+\lambda_{2}^{\prime}\right) \cdots \mathbf{L}_{a_{p}}\left(z+\lambda_{p}^{\prime}\right)=\mathcal{S}_{I} \mathbf{L}_{I}^{+}\left(z \mid \Lambda_{p}\right) \mathbb{G}_{I} \mathcal{S}_{I}^{-1}
$$

where the matrices $\mathcal{S}_{I}$ and $\mathbb{G}_{I}$ are products of the expressions of types (3.8) and (3.11) arising from the repeated use of the formula (3.4). In the particular case $p=n$ the last formula provides the factorization for the $\mathbf{L}$-operator (2.6),

$$
\mathcal{L}^{+}\left(z \mid \Lambda_{n}\right)=\pi_{\Lambda_{n}}^{+}[\mathcal{L}(z)]
$$

evaluated for the infinite-dimensional highest weight representation $\pi_{\Lambda_{n}}^{+}$in the auxiliary space,

$$
\mathbf{L}_{1}\left(z+\lambda_{1}^{\prime}\right) \mathbf{L}_{2}\left(z+\lambda_{2}^{\prime}\right) \cdots \mathbf{L}_{n}\left(z+\lambda_{n}^{\prime}\right)=\mathcal{S}_{\mathcal{L}} \mathcal{L}^{+}\left(z \mid \Lambda_{n}\right) \mathbb{G}_{\mathcal{L}} \mathcal{S}_{\mathcal{L}}^{-1}
$$

An independent proof of this fact is given in Appendix B.

\footnotetext{
6 The fact that the oscillator algebra has no finite-dimensional representation can be proved easily by taking trace of the commutation relation $\left[\mathbf{b}, \mathbf{b}^{\dagger}\right]=1$. If $\mathbf{b}, \mathbf{b}^{\dagger}$ are represented by finite-dimensional matrices the trace of the LHS vanishes while the RHS gives the dimension of the representation, leading to a contradiction.
} 


\section{Construction of the Q-operators}

The purpose of this section is to define the $\mathbf{T}$ - and $\mathbf{Q}$-operators. They have to commute with the Hamiltonian (1.1) of the twisted compact $\mathfrak{g l}(n)$-spin chain of length $L$. These operators act on the quantum space $\mathcal{V}$, which is an $L$-fold tensor product of the fundamental representations of the algebra $\mathfrak{g l}(n)$,

$$
\mathcal{V}=\underbrace{\mathbb{C}^{n} \otimes \mathbb{C}^{n} \otimes \cdots \otimes \mathbb{C}^{n}}_{L \text {-times }} .
$$

As before, solutions of the Yang-Baxter equation (2.1) are considered as $n$ by $n$ matrices, acting in the quantum space of a single spin. Their matrix elements are operators in some representation space $V$ of the Yangian algebra (2.3). This representation space will be called here the auxiliary space. For each solution of (2.1) one can define a transfer matrix,

$$
\mathbb{T}_{V}(z)=\operatorname{Tr}_{V}\{\mathbb{D} \mathbb{L}(z) \otimes \mathbb{L}(z) \otimes \cdots \otimes \mathbb{L}(z)\},
$$

where the tensor product is taken with respect to the quantum spaces $\mathbb{C}^{n}$, while the operator product and the trace is taken with respect to the auxiliary space $V$. The quantity $\mathbb{D}$ is a "boundary twist" operator acting only in auxiliary space, i.e. it acts trivially in the quantum space. This boundary operator is completely determined by the requirement of commutativity of the transfer matrix (4.2) with the Hamiltonian (1.1), which leads to the following conditions

$$
\mathbb{D}(\mathbb{L}(z))_{a b} \mathbb{D}^{-1}=e^{i\left(\Phi_{b}-\Phi_{a}\right)}(\mathbb{L}(z))_{a b}, \quad a, b=1, \ldots, n,
$$

where the fields $\Phi_{a}$ are the same as in (1.2). Note that these fields enter (4.3) only through their differences. It is convenient to set

$$
\sum_{a=1}^{n} \Phi_{a}=0
$$

Solving (4.3) for the general $\mathbf{L}$-operator (2.20) with an arbitrary set $I=\left\{a_{1}, a_{2}, \ldots, a_{p}\right\}$, one obtains

$$
\mathcal{D}_{I}=\exp \left\{i \sum_{a \in I} \Phi_{a} \mathcal{J}_{a a}-i \sum_{a \in I} \sum_{\dot{b} \notin I}\left(\Phi_{a}-\Phi_{\dot{b}}\right) \mathbf{b}_{a \dot{b}}^{\dagger} \mathbf{b}_{\dot{b} a}\right\} .
$$

Recall that $\mathcal{D}_{I}$ is related to $\mathbb{D}$ in the same way as $\mathbf{L}_{I}$ is related to $\mathbb{L}$.

In the following we will use some important properties of the trace over the Fock representations of the oscillator algebra

$$
\left[\mathbf{b}, \mathbf{b}^{\dagger}\right]=1, \quad[\mathbf{h}, \mathbf{b}]=-\mathbf{b}, \quad\left[\mathbf{h}, \mathbf{b}^{\dagger}\right]=\mathbf{b}^{\dagger}, \quad \mathbf{h}=\mathbf{b}^{\dagger} \mathbf{b}+\frac{1}{2} .
$$

This algebra has two Fock representations,

$$
\mathcal{F}_{+}: \quad \mathbf{b}|0\rangle=0, \quad|k+1\rangle=\mathbf{b}^{\dagger}|k\rangle,
$$

and

$$
\mathcal{F}_{-}: \quad \mathbf{b}^{\dagger}|0\rangle=0, \quad|k+1\rangle=\mathbf{b}|k\rangle,
$$

spanned on the vectors $|k\rangle, k=0,1,2, \ldots, \infty$. These representations can be obtained from each other via a simple automorphism of (4.6), 


$$
\mathbf{b} \rightarrow-\mathbf{b}^{\dagger}, \quad \mathbf{b}^{\dagger} \rightarrow \mathbf{b}, \quad \mathbf{h} \rightarrow-\mathbf{h} .
$$

Let $P\left(\mathbf{b}, \mathbf{b}^{\dagger}\right)$ be an arbitrary polynomial of the operators $\mathbf{b}$ and $\mathbf{b}^{\dagger}$. Below it will be convenient to use a normalized trace over the representations $\mathcal{F}_{ \pm}$,

$$
\widehat{\operatorname{Tr}}_{\mathcal{F}}\left\{e^{i \Phi \mathbf{h}} P\left(\mathbf{b}, \mathbf{b}^{\dagger}\right)\right\} \stackrel{\text { def }}{=} \frac{\operatorname{Tr}_{\mathcal{F}}\left\{e^{i \Phi \mathbf{h}} P\left(\mathbf{b}, \mathbf{b}^{\dagger}\right)\right\}}{\operatorname{Tr}_{\mathcal{F}}\left\{e^{i \Phi \mathbf{h}}\right\}}, \quad \mathcal{F}=\mathcal{F}_{ \pm},
$$

where $\mathcal{F}$ is either $\mathcal{F}_{+}$or $\mathcal{F}_{-}$, and $\operatorname{Tr}_{\mathcal{F}}$ denotes the standard trace. An important feature of the normalized trace (4.10) is that it is completely determined by the commutation relations (4.6) and the cyclic property of the trace. It is therefore independent of a particular choice of representation as long as the traces in the RHS of (4.10) converge. Alternatively, one can reproduce the same result by using explicit expressions for the matrix elements of the oscillator operators in (4.10). Then the trace over $\mathcal{F}_{+}$converges when $\operatorname{Im} \Phi>0$ and the trace over $\mathcal{F}_{-}$when $\operatorname{Im} \Phi<0$. Both ways of calculation lead to the same analytic expression for the normalized trace. Thus it is not necessary to specify which of the two representations $\mathcal{F}_{ \pm}$is used.

We are now ready to define various transfer matrices all commuting with the Hamiltonian (1.1). Consider the most general $\mathbf{L}$-operator (2.20) with an arbitrary set $I=\left\{a_{1}, a_{2}, \ldots, a_{p}\right\}$, where $p=1,2, \ldots, n$. Recall that the matrix elements of (2.20) belong to the direct product (2.16) of the algebra $\mathfrak{g l}(p)$ and of $p(n-p)$ oscillator algebras. Choose a finite-dimensional representation $\pi_{\Lambda_{p}}$ with the highest weight $\Lambda_{p}$ for the $\mathfrak{g l}(p)$-factor of this product. Then substituting (2.20) and (4.5) into (4.2) one can define rather general transfer matrices

$$
\mathbf{X}_{I}\left(z, \Lambda_{p}\right)=e^{i z\left(\sum_{a \in I} \Phi_{a}\right)} \operatorname{Tr}_{\pi_{\Lambda_{p}}} \widehat{\operatorname{Tr}}_{\mathcal{F} p(n-p)}\left\{\mathbf{M}_{I}(z)\right\},
$$

where $\mathbf{M}_{I}(z)$ is the corresponding monodromy matrix,

$$
\mathbf{M}_{I}(z)=\mathcal{D}_{I} \mathbf{L}_{I}(z) \otimes \mathbf{L}_{I}(z) \otimes \cdots \otimes \mathbf{L}_{I}(z) .
$$

Here $\widehat{\operatorname{Tr}}_{\mathcal{F} p(n-p)}$ denotes the normalized trace (4.10) for all involved oscillator algebras ${ }^{7}$ while $\operatorname{Tr}_{\pi_{\Lambda_{p}}}$ denotes the standard trace over the representation $\pi_{\Lambda_{p}}$ of $\mathfrak{g l}(p)$. The exponential scalar factor in front of the trace is introduced for later convenience.

Similarly one can define a related quantity where the $\mathfrak{g l}(p)$-trace is taken over an infinitedimensional highest weight representation $\pi_{\Lambda_{p}}^{+}$,

$$
\mathbf{X}_{I}^{+}\left(z, \Lambda_{p}\right)=e^{i z\left(\sum_{a \in I} \Phi_{a}\right)} \operatorname{Tr}_{\pi_{\Lambda_{p}}^{+}} \widehat{\operatorname{Tr}}_{\mathcal{F} p(n-p)}\left\{\mathbf{M}_{I}(z)\right\}
$$

\footnotetext{
7 It is easy to check that all possible expressions under the trace in (4.11) for each oscillator algebra are exactly as in the LHS of (4.10) for some polynomial $P$ and some value of $\Phi$. Thus the definition (4.10) is sufficient to calculate all oscillator traces in (4.11).

We would like to stress that the possibility to define a non-trivial trace over representations of the algebra (2.14), suitable for the definition of the transfer matrix (4.2) with twisted boundary conditions, heavily relies on the fact that the matrix $D_{\dot{a} \dot{b}}$ in (2.14) is non-degenerate. Indeed, if this non-degeneracy condition is not met then the algebra (2.14) would contain at least one $\mathfrak{e}(2)$ sub-algebra. The later is generated by three elements $\boldsymbol{h}, \boldsymbol{b}, \boldsymbol{b}^{*}$, obeying the relations
}

$$
\mathfrak{e}(2): \quad\left[\boldsymbol{b}, \boldsymbol{b}^{*}\right]=0, \quad[\boldsymbol{h}, \boldsymbol{b}]=-\boldsymbol{b}, \quad\left[\boldsymbol{h}, \boldsymbol{b}^{*}\right]=\boldsymbol{b}^{*} .
$$

Proceeding as above with the construction of the transfer matrix one would come across to the necessity of defining traces of the form $\operatorname{Tr}\left\{e^{i \Phi \boldsymbol{h}} P\left(\boldsymbol{b}, \boldsymbol{b}^{*}\right)\right\}$. However, for a non-zero field $\Phi$, most of these traces must immediately vanish by virtue of the cyclic property of the trace and the above commutation relations of the $\mathfrak{e}(2)$ algebra. For instance, using these relations one can easily show that

$$
\operatorname{Tr}\left\{e^{i \Phi \boldsymbol{h}}\left(\boldsymbol{b} \boldsymbol{b}^{*}\right)^{k}\right\}=e^{i k \Phi} \operatorname{Tr}\left\{e^{i \Phi \boldsymbol{h}}\left(\boldsymbol{b} \boldsymbol{b}^{*}\right)^{k}\right\} \quad \Rightarrow \quad \operatorname{Tr}\left\{e^{i \Phi \boldsymbol{h}}\left(\boldsymbol{b} \boldsymbol{b}^{*}\right)^{k}\right\} \equiv 0, \quad \text { if } \Phi \neq 0, k \geqslant 1 .
$$


while the rest of the expression remains the same as in (4.11). Note that in the case of (4.11) the weights $\Lambda_{p}=\left(\lambda_{1}, \lambda_{2}, \ldots, \lambda_{p}\right)$ satisfy the conditions (2.25). In contradistinction, in (4.13) these weights are arbitrary.

In the limiting case $p=n$, the general $\mathbf{L}$-operator (2.20) simplifies to (2.6), while the expression (4.5) simplifies to

$$
\mathcal{D}=\mathcal{D}_{\{1,2, \ldots, n\}}=\exp \left\{i \sum_{a=1}^{n} \Phi_{a} \mathcal{J}_{a a}\right\} .
$$

In this case the definition (4.11) reduces to that for the standard $\mathbf{T}$-operator

$$
\mathbf{T}_{\Lambda_{n}}(z) \equiv \mathbf{X}_{\{1,2, \ldots, n\}}\left(z, \Lambda_{n}\right)=\operatorname{Tr}_{\pi_{\Lambda_{n}}}\{\mathcal{D} \mathcal{L}(z) \otimes \mathcal{L}(z) \otimes \cdots \otimes \mathcal{L}(z)\}
$$

associated with the finite-dimensional representation $\pi_{\Lambda_{n}}$ of the algebra $\mathfrak{g l}(n)$ in the auxiliary space. Here $\mathcal{L}(z)$ denotes the $\mathbf{L}$-operator (2.6). Likewise, the formula (4.13) reduces to the $\mathbf{T}$ operator

$$
\mathbf{T}_{\Lambda_{n}}^{+}(z)=\mathbf{X}_{\{1,2, \ldots, n\}}^{+}\left(z, \Lambda_{n}\right)
$$

associated with the infinite-dimensional representation $\pi_{\Lambda_{n}}^{+}$. The above two $\mathbf{T}$-operators are connected due the Bernstein, Gel'fand and Gel'fand (BGG) resolution of the finite-dimensional modules [35]. The BGG result allows one to express finite-dimensional highest weight modules in terms of an alternating sum of infinite-dimensional highest weight modules. This implies that the $\mathbf{T}$-operator (4.15) for a finite-dimensional module can be written in terms of (4.16) as

$$
\mathbf{T}_{\Lambda_{n}}(z)=\sum_{\sigma \in S_{n}}(-1)^{l(\sigma)} \mathbf{T}_{\sigma\left(\Lambda_{n}+\rho_{n}\right)-\rho_{n}}^{+}(z),
$$

where $\rho_{n}$ is a constant $n$-component vector

$$
\rho_{n}=\left(\frac{n-1}{2}, \frac{n-3}{2}, \ldots, \frac{1-n}{2}\right),
$$

which coincides with the half sum of the positive roots of the algebra $\mathfrak{s l}(n)$. The summation in (4.17) is taken over all permutations of $n$ elements, $\sigma \in S_{n}$, and $l(\sigma)$ is the parity of the permutation $\sigma$. The relation (4.17) and its connection to the BGG resolution were first obtained in [19] in the context of $U_{q}(\widehat{s l}(3))$, while the $n=2$ case was previously considered in [6,22].

Similarly, for (4.11) one has

$$
\mathbf{X}_{I}\left(z, \Lambda_{p}\right)=\sum_{\sigma \in S_{p}}(-1)^{l(\sigma)} \mathbf{X}_{I}^{+}\left(z, \sigma\left(\Lambda_{p}+\rho_{p}\right)-\rho_{p}\right),
$$

where $\rho_{p}$ is a $p$-component vector defined as in (4.18) with $n$ replaced by $p$.

Another limiting case of (4.11) corresponds to the representation $\pi_{\Lambda_{p}}$ turning into the trivial one-dimensional representation of $\mathfrak{g l}(p)$ with weight $\Lambda=(0,0, \ldots, 0)$. As we shall see below the resulting operators

$$
\mathbf{Q}_{I}(z)=\mathbf{X}_{I}(z,(0))
$$

are actually the $\mathbf{Q}$-operators, whose eigenvalues appear in the nested Bethe Ansatz equations. Let us enumerate these $\mathbf{Q}$-operators. It is convenient to start formally from the exceptional case $p=0$, corresponding to an empty set $I=\varnothing$. By definition we set 


$$
\mathbf{Q}_{\varnothing}(z) \equiv 1 .
$$

For the next level $p=1$ there are obviously $n$ sets $I$ consisting of just one element $I=\{a\}$, $a=1,2, \ldots, n$. The general $\mathbf{L}$-operator (2.20) in this case takes the simple form (1.16) and the twist operator (4.5) simplifies to

$$
\mathcal{D}_{a} \equiv \mathcal{D}_{\{a\}}=\exp \left\{-i \sum_{\dot{c} \notin I}\left(\Phi_{a}-\Phi_{\dot{c}}\right) \mathbf{b}_{a \dot{c}}^{\dagger} \mathbf{b}_{\dot{c} a}\right\}, \quad a=1,2, \ldots, n .
$$

In this way one obtains from (4.11)

$$
\mathbf{Q}_{a}(z) \equiv \mathbf{Q}_{\{a\}}(z)=\mathbf{X}_{\{a\}}(z,(0))=e^{i z \Phi_{a}} \widehat{\operatorname{Tr}}_{\mathcal{F}^{(n-1)}}\left\{\mathcal{D}_{a} \mathbf{L}_{a}(z) \otimes \mathbf{L}_{a}(z) \otimes \cdots \otimes \mathbf{L}_{a}(z)\right\}
$$

where $a=1,2, \ldots, n$ and $\mathbf{L}_{a}(z)$ is given by (1.16).

More generally, for the level $p$ there are $\left(\begin{array}{l}n \\ p\end{array}\right)$ increasing integer sets $I=\left\{a_{1}, \ldots, a_{p}\right\} \subseteq$ $\{1,2, \ldots, n\}$, which numerate the $\mathbf{Q}$-operators (4.20). For the highest level $p=n$ the definitions (4.20) and (4.11) immediately lead to the result

$$
\mathbf{Q}_{\{1,2, \ldots, n\}}(z)=z^{L},
$$

where $L$ is the length of the chain. Altogether there are $2^{n}$ different $\mathbf{Q}$-operators, ${ }^{8}$ including (4.21) and (4.24). These operators form in conjunction with all $\mathbf{T}$ - and $\mathbf{X}$-operators a commuting family and therefore can be simultaneously diagonalized. It is easy to see that their eigenvalues have the form

$$
\mathrm{Q}_{I}(z)=e^{i z\left(\sum_{a \in I} \Phi_{a}\right)} \prod_{k=1}^{m_{I}}\left(z-z_{k}^{I}\right), \quad m_{I}=\sum_{a \in I} m_{a},
$$

where, for each eigenstate, the numbers $m_{a}$ are the conserved occupation numbers,

$$
m_{1}+m_{2}+\cdots+m_{n}=L
$$

defined in the Introduction.

We would like to stress, that in general the operators $\mathbf{X}_{I}\left(z, \Lambda_{p}\right)$, defined in (4.11), involve the trace over a representation of the Lie algebra $\mathfrak{g l}(p)$ and the trace over a number of Fock representations of the oscillator algebra, whereas the $\mathbf{T}$-operators involve only the Lie algebra trace and the Q-operator only the oscillator traces. This is why we denoted the "hybrid" operators (4.11) by a distinct symbol $\mathbf{X}$ (thereby continuing a steady tradition of the field which already have Y-systems, T-systems, Q-systems, etc., to occupy all letters of the alphabet).

\section{Functional relations}

The results of Section 3 imply various functional relations for the $\mathbf{Q}$-operators. To derive them we need to use some additional properties of the twist operators (4.5) which are not immediately obvious from their definition (4.3). Let $\mathcal{D}_{I}$ and $\mathcal{D}_{J}$ be the operators (4.5), corresponding to $\mathbf{L}_{I}^{[1]}(z)$ and $\mathbf{L}_{J}^{[2]}(z)$ from the LHS of (3.4). By explicit calculation one can check that the product of these operators commutes with the similarity transformation $\mathcal{S}$ defined in (3.8),

\footnotetext{
8 The Q-operators can be conveniently associated with nodes of a hypercubical Hasse diagram. They are labelled by their index sets $I=\left\{a_{1}, a_{2}, \ldots, a_{p}\right\}$, ordered by inclusion. See next section, and Appendix C.
} 


$$
\left[\mathcal{D}_{I} \mathcal{D}_{J}, \mathcal{S}\right]=0
$$

Moreover this product can be rewritten in the form

$$
\mathcal{D}_{I} \mathcal{D}_{J}=\mathcal{D}_{I \cup J} \mathcal{D}_{\mathbb{G}}
$$

where $\mathcal{D}_{I \cup J}$ and $\mathcal{D}_{\mathbb{G}}$ are the twist operators obtained from (4.3) for the operator $\mathbf{L}_{I \cup J}(z)$ and the $z$-independent $\mathbf{L}$-operator $\mathbb{G}$ from the RHS of (3.4). Again the relation (5.2) is verified by direct calculation, where one needs to take into account the explicit form of (3.11), (3.12), (3.13) and (3.14).

Next, define a scalar factor, cf. (1.4),

$$
\Delta_{I}(\Phi)=\Delta_{\left\{a_{1}, a_{2}, \ldots, a_{p}\right\}}(\Phi)=\prod_{1 \leqslant i<j \leqslant p} 2 i \sin \left(\frac{\Phi_{a_{i}}-\Phi_{a_{j}}}{2}\right),
$$

which depends on the set $I$ and the fields $\Phi_{1}, \Phi_{2}, \ldots, \Phi_{n}$. Combining (3.4) with the definition (4.13) and taking into account (5.1) and (5.2) one obtains

$$
\Delta_{I} \mathbf{X}_{I}^{+}\left(z+\frac{p_{2}}{2}, \Lambda_{p_{1}}\right) \Delta_{J} \mathbf{X}_{J}^{+}\left(z+\lambda-\frac{p_{1}}{2}, \Lambda_{p_{2}}\right)=\Delta_{I \cup J} \mathbf{X}_{I \cup J}^{+}\left(z, \Lambda_{p_{1}+p_{2}}\right) .
$$

There are two nontrivial steps in the derivation of the last formula which require explanations. First, the simple transfer matrix

$$
\mathbf{T}_{\mathbb{G}}=\widehat{\operatorname{Tr}}_{\mathcal{F} p_{1} p_{2}}\left\{\mathbb{D}_{\mathbb{G}} \mathbb{G} \otimes \mathbb{G} \otimes \cdots \otimes \mathbb{G}\right\}=1
$$

that arises in the calculations is equal to the identity operator. Second, the scalar factors in (5.4) arise due to the difference in the definition of the trace over the oscillator algebras (normalized trace (4.10)) and over the representation of $\mathfrak{g l}(p)$ (standard trace). From (3.15) it is clear that $p_{1} p_{2}$ oscillator pairs have to be included in the definition of the generators in (3.12) in order to support the Holstein-Primakoff realization of the infinite-dimensional representation $\pi_{\Lambda_{p_{1}+p_{2}}}^{+}$of the algebra $\mathfrak{g l}\left(p_{1}+p_{2}\right)$.

A particular simple case of (5.4) arises when $p_{2}=1$ and $J=\left\{a_{p+1}\right\}$,

$$
\Delta_{I} \mathbf{X}_{I}^{+}\left(z+\frac{1}{2}, \Lambda_{p}\right) \mathbf{Q}_{a_{p+1}}\left(z+\lambda_{p+1}-\frac{p}{2}\right)=\Delta_{I \cup a_{p+1}} \mathbf{X}_{I \cup a_{p+1}}^{+}\left(z, \Lambda_{p+1}\right),
$$

where by the definition (4.23) one has $\mathbf{Q}_{a}(z) \equiv \mathbf{X}_{\{a\}}(z,(0))$. Iterating the last formula one obtains

$$
\Delta_{I} \mathbf{X}_{I}^{+}\left(z, \Lambda_{p}\right)=\mathbf{Q}_{a_{1}}\left(z+\lambda_{1}^{\prime}\right) \mathbf{Q}_{a_{2}}\left(z+\lambda_{2}^{\prime}\right) \cdots \mathbf{Q}_{a_{p}}\left(z+\lambda_{p}^{\prime}\right),
$$

where the notation here is the same as in (3.19) and (3.20). Next, applying (4.19) one gets

$$
\Delta_{I} \mathbf{X}_{I}\left(z, \Lambda_{p}\right)=\operatorname{det}\left\|\mathbf{Q}_{a_{i}}\left(z+\lambda_{j}^{\prime}\right)\right\|_{1 \leqslant i, j \leqslant p},
$$

and setting $\Lambda_{p}=(0)$ one finally arrives at

$$
\Delta_{I} \mathbf{Q}_{I}(z)=\operatorname{det}\left\|\mathbf{Q}_{a_{i}}\left(z-j+\frac{p+1}{2}\right)\right\|_{1 \leqslant i, j \leqslant p} .
$$

Note also that in a particular case $I=\{1,2, \ldots, n\}$ the formula (5.8) leads to the determinant expression $[6,17,22,19,24]$ for transfer matrix (4.15),

$$
\Delta_{\{1,2, \ldots, n\}} \mathbf{T}_{\Lambda_{n}}(z)=\operatorname{det}\left\|\mathbf{Q}_{i}\left(z+\lambda_{j}^{\prime}\right)\right\|_{1 \leqslant i, j \leqslant n},
$$

where $\Delta_{\{\ldots\}}$ is defined in (5.3) and $\lambda_{j}^{\prime}$ in (3.19). 

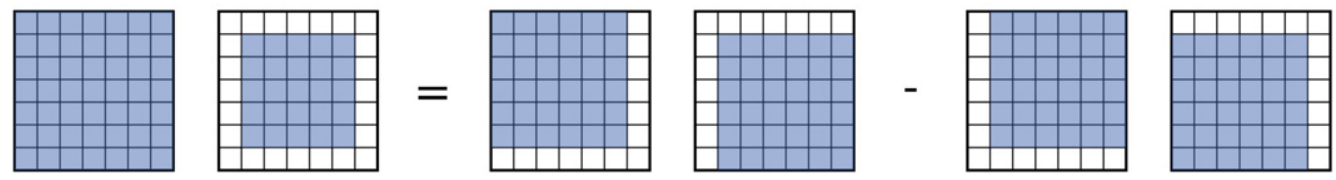

Fig. 2. Jacobi determinant formula.

As previously mentioned the $2^{n}$ different operators $\mathbf{Q}_{I}$ can be assigned to the nodes of a hypercubic Hasse diagram. We will now show that four Q-operators belonging to the same quadrilateral as in Fig. 5 satisfy a remarkably simple functional equation, which can be identified with the famous Hirota equation from the theory of classical discrete evolution equation. Define a matrix

$$
M_{i j} \equiv \mathbf{Q}_{a_{i}}\left(z-j+\frac{p+1}{2}\right), \quad i, j \in\{0, \ldots, p+1\},
$$

where $\left\{a_{0}, a_{1}, \ldots, a_{p}, a_{p+1}\right\}$ in an increasing sequence of $p+2$ integers which contains the subsequence $I=\left\{a_{1}, \ldots, a_{p}\right\}$. Denote $a \equiv a_{0}$ and $b \equiv a_{p+1}$. Let us now use Jacobi's formula for determinants (see Fig. 2) for the matrix (5.11). Applying (5.9) for the subdeterminants one obtains the following operatorial functional relation ${ }^{9}$

$$
\Delta_{\{a, b\}} \mathbf{Q}_{I \cup a \cup b}(z) \mathbf{Q}_{I}(z)=\mathbf{Q}_{I \cup a}\left(z-\frac{1}{2}\right) \mathbf{Q}_{I \cup b}\left(z+\frac{1}{2}\right)-\mathbf{Q}_{I \cup b}\left(z-\frac{1}{2}\right) \mathbf{Q}_{I \cup a}\left(z+\frac{1}{2}\right),
$$

which was already stated in the introduction as (1.3), and where $\Delta_{a, b}$ is given in (1.4). Since all Q-operators commute with each other the same relation (5.12) holds also for the corresponding eigenvalues. We will use it to derive Bethe equations in the next section.

\section{Bethe equations}

The connection between the Hirota equations (5.12) and the Bethe Ansatz equations is well understood [6,18-20,36,16]. Consider Eq. (5.12) at the eigenvalue level. The reader might find it useful to look at the examples of Hasse diagrams in the introduction and in Appendix $\mathrm{C}$ when following through the following derivation. Let us denote the zeroes of $\mathrm{Q}_{I \cup a}(z)$ by $z_{k}^{I \cup a}$. Taking $z+\frac{1}{2}=z_{k}^{I \cup a}$ and $z-\frac{1}{2}=z_{k}^{I \cup a}$, Eq. (5.12) reads

$$
\begin{aligned}
& \mathrm{Q}_{I \cup a \cup b}\left(z_{k}^{I \cup a}-\frac{1}{2}\right) \mathrm{Q}_{I}\left(z_{k}^{I \cup a}-\frac{1}{2}\right) \sim \mathrm{Q}_{I \cup a}\left(z_{k}^{I \cup a}-1\right) \mathrm{Q}_{I \cup b}\left(z_{k}^{I \cup a}\right), \\
& \mathrm{Q}_{I \cup a \cup b}\left(z_{k}^{I \cup a}+\frac{1}{2}\right) \mathrm{Q}_{I}\left(z_{k}^{I \cup a}+\frac{1}{2}\right) \sim-\mathrm{Q}_{I \cup a}\left(z_{k}^{I \cup a}+1\right) \mathrm{Q}_{I \cup b}\left(z_{k}^{I \cup a}\right),
\end{aligned}
$$

respectively, where $a, b \notin I$. Taking the ratio of these two equations above one obtains

$$
-1=\frac{\mathrm{Q}_{I}\left(z_{k}^{I \cup a}-\frac{1}{2}\right)}{\mathrm{Q}_{I}\left(z_{k}^{I \cup a}+\frac{1}{2}\right)} \frac{\mathrm{Q}_{I \cup a}\left(z_{k}^{I \cup a}+1\right)}{\mathrm{Q}_{I \cup a}\left(z_{k}^{I \cup a}-1\right)} \frac{\mathrm{Q}_{I \cup a \cup b}\left(z_{k}^{I \cup a}-\frac{1}{2}\right)}{\mathrm{Q}_{I \cup a \cup b}\left(z_{k}^{I \cup a}+\frac{1}{2}\right)} .
$$

Here $I$ can also be the empty set. In this case we can remove $\mathrm{Q}_{\varnothing}(z)$ from the equation using (4.21). The number of elements in $I \cup a \cup b$ cannot exceed $n$, therefore $I$ contains at most $n-2$ elements. Thus one obtains $n-1$ different relations of the type (6.3), with various cardinalities of the set $I$. This exactly matches the number of levels of nested Bethe equations for the $\mathfrak{g l}(n)$-spin chain.

\footnotetext{
9 More general relations involving operators (5.8) can be obtained in the same way by replacing the arguments of the Q-operators in (5.11) with arbitrary constants $z_{j}, j=0, \ldots, p+1$.
} 
Let us take any sequence $\left(a_{1}, \ldots, a_{n}\right)$ of elements of the set $\{1, \ldots, n\}$. We construct a sequence of ascending sets $\varnothing=I_{0} \subset I_{1} \subset \cdots \subset I_{n}=\{1, \ldots, n\}$ such that ${ }^{10} I_{i}=I_{i-1} \cup a_{i}$. Then for each $I_{i}, i=1, \ldots, n-1$, we can rewrite $(6.3)$ as

$$
-1=\frac{\mathrm{Q}_{I_{i-1}}\left(z_{k}^{I_{i}}-\frac{1}{2}\right)}{\mathrm{Q}_{I_{i-1}}\left(z_{k}^{I_{i}}+\frac{1}{2}\right)} \frac{\mathrm{Q}_{I_{i}}\left(z_{k}^{I_{i}}+1\right)}{\mathrm{Q}_{I_{i}}\left(z_{k}^{I_{i}}-1\right)} \frac{\mathrm{Q}_{I_{i+1}}\left(z_{k}^{I_{i}}-\frac{1}{2}\right)}{\mathrm{Q}_{I_{i+1}}\left(z_{k}^{I_{i}}+\frac{1}{2}\right)} .
$$

We will call an equation with $z^{I_{i}}$ roots the $i$-th level equation. One need to use (4.21) for the lowest level, $i=1$, and (4.24) for the highest level equation with $i=n-1$.

It is not difficult to see that the system of functional equations (6.3) already corresponds to the nested system of Bethe equations of the $\mathfrak{g l}(n)$ compact magnets. To recover their traditional form, we merely need to substitute the eigenvalue formula (4.25) into (6.4). The crucial point of our approach is that the analytic structure of (4.25) is rigorously derived without any assumptions. It immediately follows from the explicit construction of the $\mathbf{Q}$-operators in Section 4. Then one can rewrite (6.4) as

$$
e^{i\left(\Phi_{a_{2}}-\Phi_{a_{1}}\right)}=\prod_{k \neq l} \frac{z_{l}^{I_{1}}-z_{k}^{I_{1}}+1}{z_{l}^{I_{1}}-z_{k}^{I_{1}}-1} \prod_{k} \frac{z_{l}^{I_{1}}-z^{I_{2}}-\frac{1}{2}}{z_{k}^{I_{1}}-z^{I_{2}}+\frac{1}{2}},
$$

for the lowest level,

$$
e^{i\left(\Phi_{a_{i+1}}-\Phi_{a_{i}}\right)}=\prod_{k} \frac{z_{l}^{I_{i}}-z_{k}^{I_{i-1}}-\frac{1}{2}}{z_{l}^{I_{i}}-z_{k}^{I_{i-1}}+\frac{1}{2}} \prod_{k \neq l} \frac{z_{l}^{I_{i}}-z_{k}^{I_{i}}+1}{z_{l}^{I_{i}}-z_{k}^{I_{i}}-1} \prod_{k} \frac{z_{l}^{I_{i}}-z^{I_{i+1}}-\frac{1}{2}}{z_{k}^{I_{i}}-z^{I_{i+1}}+\frac{1}{2}},
$$

for $i=2, \ldots, n-2$ and for the highest level

$$
e^{i\left(\Phi_{a_{n}}-\Phi_{a_{n-1}}\right)}\left(\frac{z_{l}^{I_{n-1}}+\frac{1}{2}}{z_{l}^{I_{n-1}}-\frac{1}{2}}\right)^{L}=\prod_{k} \frac{z_{l}^{I_{n-1}}-z_{k}^{I_{n-2}}-\frac{1}{2}}{z_{l}^{I_{n-1}}-z_{k}^{I_{n-2}}+\frac{1}{2}} \prod_{k \neq l} \frac{z_{l}^{I_{n-1}}-z_{k}^{I_{n-1}}+1}{z_{l}^{I_{n-1}}-z_{k}^{I_{n-1}}-1} .
$$

Eqs. (6.5)-(6.7) are exactly the Bethe equations for the compact $\mathfrak{g l}(n)$ symmetric spin chain. It is obvious that there are $n$ ! alternative forms of the above Bethe Ansatz equations, corresponding to $n$ ! permutations of the elements of the set $I$, which in turn are associated with the $n$ ! different bottom-to-top paths on the Hasse diagram.

To conclude our new solution procedure for the $\mathfrak{g l}(n)$-spin chain we give the expression for the eigenvalues of (1.1). It only involves the roots $z^{I_{n-1}}$ on the last-level

$$
E=2 \sum_{k=1}^{m_{I_{n-1}}} \frac{1}{\frac{1}{4}-\left(z_{k}^{I_{n-1}}\right)^{2}}
$$

where $m_{I_{n-1}}$ is the number of roots of the eigenvalue $Q_{I_{n-1}}(z)$, which according to (4.25) is equal to

$$
m_{I_{n-1}}=m_{a_{1}}+m_{a_{2}}+\cdots+m_{a_{n-1}}=L-m_{a_{n}} .
$$

The derivation of the energy (6.8) from the functional relations is given in Appendix D.

$\overline{10}$ This construction can easily be obtained by choosing a path on the Hasse diagram leading from $\varnothing$ to $\{1, \ldots, n\}$. See Appendix C for more details. 


\section{Conclusions and outlook}

In this paper we developed a novel, systematic procedure for constructing the Baxter Qoperators connected with the $\mathfrak{g l}(n)$-spin chain (1.1) with quasi-periodic boundary conditions. This fills an important gap in the literature: $\mathbf{Q}$-operators have been originally designed to solve quantum integrable models for which no suitable pseudovacuum exists, a pre-condition for the application of Bethe's Ansatz. They have furthermore mostly been constructed for cases where the rank of the symmetry algebra is one. In order to develop a universal theory of the $\mathbf{Q}$-operator it therefore seems indispensable to fill the gap and construct these operators also in the cases where the Bethe Ansatz does work, and to generalize to arbitrary ranks of the symmetry algebra. For illustration purposes we confined ourselves to the case where the quantum space is a multiple tensor product of the compact, unitary, fundamental representation of $\mathfrak{s u}(n)$. The $\mathbf{Q}$-operators are constructed as transfer matrices associated with infinite-dimensional representations of the Yangian $Y(\mathfrak{g l}(n))$ built from Fock representations of the harmonic oscillator algebra. This involves rather simple, but hitherto unknown solutions (2.20) of the Yang-Baxter equations with first order dependence on the spectral parameter. These solutions provide fundamental building blocks for all other required solutions via the standard fusion procedure. As a result we derived the full set of functional relations, which enabled us to obtain a new algebraic solution of the $\mathfrak{g l}(n)$-spin chain independent of the Bethe Ansatz.

The reader might wonder why we have confined ourselves in our search for novel solutions of the Yang-Baxter equation to truncating the series in (2.4) after the second term, i.e. evaluation representation. The answer is that this is fully sufficient in order to treat the $\mathfrak{g l}(n)$-spin chains studied in this paper. Moreover, having in mind previous results about representations of Yangians $[32,33,26]$, we suspect that taking into account higher terms in (2.4) will not lead to new solutions not obtainable by fusion from the ones of the form (2.12). However, it would certainly be interesting to investigate this issue in some detail.

The construction we have presented in this paper generalizes in a beautiful fashion to compact spin chains with $\mathfrak{g l}(n \mid m)$ supersymmetry. In particular, the partonic Lax operators (1.16) naturally generalize to the supersymmetric case. Details on this will be reported elsewhere [12].

Our methodology also generalizes to more general representations in the quantum space: Recall that in this paper we always took it to be a tensor product of $L$ fundamental $n$-dimensional representations. Particularly relevant is the case of non-compact spin chains. It will be interesting to spell out the exact relation between our construction of $\mathbf{Q}$-operators and a rather different approach proposed in [37] and a large number of earlier work cited therein.

\section{Acknowledgements}

We thank Volodya Kazakov, Zengo Tsuboi and Changrim Ahn for useful discussions. One of us (V.B.) thanks Alexander Molev and Sergei Sergeev for very illuminating discussions. When finishing this paper, we became aware of [38], which also proposes an (apparently quite different) method for constructing $\mathbf{Q}$-operators for compact $\mathfrak{g l}(n)$-spin chains, based on the co-derivative formalism [39]. We would like to thank the Institute for the Early Universe at Ewha Womans University in Seoul, the Center for Quantum Spacetime at Sogang University in Seoul, and the APCTP in Pohang for hospitality during the completion phase of this work. T. Łukowski is supported by a DFG grant in the framework of the SFB 647 "Raum-Zeit-Materie. Analytische und Geometrische Strukturen" and by Polish science funds during 2009-2011 as a research project (NN202 105136). 


\section{Appendix A. Fusion of the canonical L-operators}

The calculations which one has to perform in order to prove formula (3.4) are tedious but rather straightforward. It is enough to calculate the matrix product of the operator-valued matrices (3.6) and (3.7), and subsequently use the similarity transforms (3.9) and (3.10), keeping in mind the commutation relations between the various oscillators. For the convenience of the reader we present here the explicit action of these similarity transforms $\mathcal{S}_{1}$ and $\mathcal{S}_{2}$ on the oscillators.

\section{A.1. Similarity transform $\mathcal{S}_{1}$}

$$
\begin{gathered}
\mathcal{S}_{1} \mathbf{b}_{\dot{a} b}^{[1]} \mathcal{S}_{1}^{-1}=\mathbf{b}_{\dot{a} b}^{[1]}-\mathbf{b}_{\dot{a} b}^{\dagger[2]}, \\
\mathcal{S}_{1} \mathbf{b}_{b \dot{a}}^{[2]} \mathcal{S}_{1}^{-1}=\mathbf{b}_{b \dot{a}}^{[2]}-\mathbf{b}_{b \dot{a}}^{\dagger[1]} .
\end{gathered}
$$

All other oscillators are unchanged.

\section{A.2. Similarity transform $\mathcal{S}_{2}$}

$$
\begin{aligned}
& \mathcal{S}_{2} \mathbf{b}_{\dot{a} b}^{[1]} \mathcal{S}_{2}^{-1}=\mathbf{b}_{\dot{a} b}^{[1]}-\sum_{\ddot{c} \notin I \cup J} \mathbf{b}_{\dot{a} \ddot{c}}^{\dagger[2]} \mathbf{b}_{\ddot{c} b}^{[1]}, \\
& \mathcal{S}_{2} \mathbf{b}_{\ddot{a} \dot{b}}^{[2]} \mathcal{S}_{2}^{-1}=\mathbf{b}_{\ddot{a} \dot{b}}^{[2]}-\sum_{c \in I} \mathbf{b}_{c \dot{b}}^{\dagger[1]} \mathbf{b}_{\ddot{a} c}^{[1]}, \\
& \mathcal{S}_{2} \mathbf{b}_{b \ddot{a}}^{\dagger[1]} \mathcal{S}_{2}^{-1}=\mathbf{b}_{b \ddot{a}}^{\dagger[1]}+\sum_{\dot{c} \in J} \mathbf{b}_{b \dot{c}}^{\dagger[1]} \mathbf{b}_{\dot{c} \ddot{a}}^{\dagger[2]}
\end{aligned}
$$

All other oscillators are unchanged.

\section{Appendix B. Proof of the factorization formula}

In this appendix we prove that the ordered product of the $n$ different partonic Lax operators, each containing $n-1$ pairs of oscillators, indeed can be disentangled by a similarity transform $\mathcal{S}_{\mathcal{L}}$ into the product of the $\mathfrak{g l}(n)$ invariant Lax operator $\mathcal{L}^{+}$and a compensating matrix $\mathbb{G}_{\mathcal{L}}$. Further details were discussed in Section 1. Instead of using the prescription developed in Section 3 to derive Eq. (1.18) inductively, we consider the following decomposition of the partonic Lax operators:

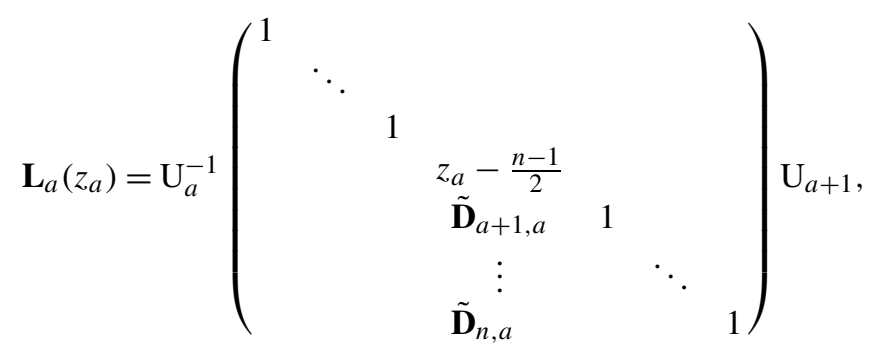

with $z_{a}=z+\lambda_{a}^{\prime}$.

The matrices $\mathrm{U}_{a}$ do not depend on the spectral parameter. They are of the form 


$$
\mathrm{U}_{a}=\left(\mathbb{I}+\sum_{1 \leqslant b<c<a} \mathbf{b}_{b c} e_{b c}\right)^{-1}\left(\mathbb{I}-\sum_{b=1}^{a-1} \sum_{c=a}^{n} \mathbf{b}_{c b}^{\dagger} e_{c b}\right)\left(\mathbb{I}-\sum_{a \leqslant b<c \leqslant n} \mathbf{b}_{b c}^{\dagger} e_{b c}\right) .
$$

The entries in the column below the spectral parameter of the middle matrix on the right-hand side of Eq. (B.1) take the values

$$
\tilde{\mathbf{D}}_{a b}=+\mathbf{b}_{a b}^{\dagger}-\mathbf{b}_{a b}+\sum_{c=1}^{b-1} \mathbf{b}_{a c}^{\dagger} \mathbf{b}_{c b}+\sum_{c=a+1}^{n} \mathbf{b}_{a c}^{\dagger} \mathbf{b}_{c b},
$$

the remaining non-diagonal entries vanish.

From this decomposition it is obvious that the product of the $n$ partonic Lax operators can be written as

$$
\mathbf{L}_{1}\left(z_{1}\right) \cdots \mathbf{L}_{n}\left(z_{n}\right)=\mathrm{U}_{1}^{-1}\left(\begin{array}{cccc}
z_{1}-\frac{n-1}{2} & & & \\
\tilde{\mathbf{D}}_{2,1} & \ddots & & \\
\vdots & \ddots & \ddots & \\
\tilde{\mathbf{D}}_{n, 1} & \cdots & \tilde{\mathbf{D}}_{n, n-1} & z_{n}-\frac{n-1}{2}
\end{array}\right) \mathrm{U}_{n+1},
$$

where two matrices $\mathrm{U}_{1}$ and $\mathrm{U}_{n+1}$ only contain an upper triangular part:

$$
\mathrm{U}_{1}=\left(\begin{array}{cccc}
1 & -\mathbf{b}_{12}^{\dagger} & \cdots & -\mathbf{b}_{1 n}^{\dagger} \\
& \ddots & \ddots & \vdots \\
& & \ddots & -\mathbf{b}_{n-1 n}^{\dagger} \\
& & & 1
\end{array}\right), \quad \mathrm{U}_{n+1}=\left(\begin{array}{cccc}
1 & \mathbf{b}_{12} & \cdots & \mathbf{b}_{1 n} \\
& \ddots & \ddots & \vdots \\
& & \ddots & \mathbf{b}_{n-1 n} \\
& & & 1
\end{array}\right)^{-1}
$$

Up to now, the lower triangular matrix in Eq. (B.4) contains all $n-1$ oscillators. A short calculation shows that half of them can be absorbed by the similarity transform $\mathcal{S}_{\mathcal{L}}=S_{n} \cdots S_{1}$ with

$$
S_{a}=\exp \left[\sum_{b=1}^{a-1}\left(\mathbf{b}_{b a}^{\dagger}+\sum_{c=b+1}^{a-1} \mathbf{b}_{c a}^{\dagger} \mathbf{b}_{b c}\right) \mathbf{b}_{a b}^{\dagger}\right] \text {. }
$$

This transformation coincides with the one that can be obtained from the method ${ }^{11}$ established in Section 3. Furthermore, it leaves $U_{1}$ invariant and its action on $U_{n+1}$ factorizes as

$$
\mathcal{S}_{\mathcal{L}} \mathrm{U}_{n+1} \mathcal{S}_{\mathcal{L}}^{-1}=\mathrm{U}_{1}^{-1} \mathrm{U}_{n+1}
$$

As a result one obtains that the ordered product of $n$ partonic Lax operators can be written as

$$
\mathbf{L}_{1}\left(z_{1}\right) \cdots \mathbf{L}_{n}\left(z_{n}\right)=\mathcal{S}_{\mathcal{L}} \mathrm{U}_{1}^{-1}\left(\begin{array}{cccc}
z_{1}-\frac{n-1}{2} & & & \\
\mathbf{D}_{2,1} & \ddots & & \\
\vdots & \ddots & \ddots & \\
\mathbf{D}_{n, 1} & \cdots & \mathbf{D}_{n, n-1} & z_{n}-\frac{n-1}{2}
\end{array}\right) \mathrm{U}_{1} \mathrm{U}_{n+1} \mathcal{S}_{\mathcal{L}}^{-1}
$$

where

\footnotetext{
11 As we are interested in the factorization formula where the compensating matrix appears on the right-hand side of the Lax operator it is convenient to start from the partonic Lax operators $\mathbf{L}_{n}\left(z_{n}\right)$ and multiply the remaining ones in the desired ordering from the left.
} 


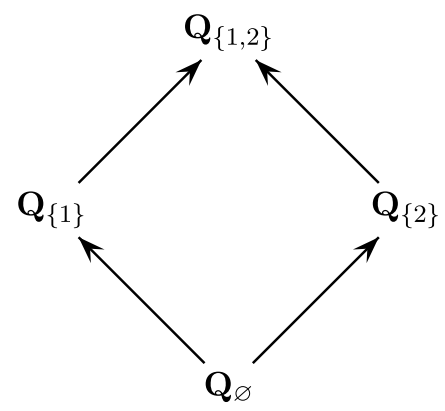

Fig. 3. Hasse diagram for $\mathfrak{g l}(2)$.

$$
\mathbf{D}_{a b}=-\mathbf{b}_{a b}+\sum_{c=a+1}^{n} \mathbf{b}_{a c}^{\dagger} \mathbf{b}_{c b} .
$$

We identify the $\mathfrak{g l}(n)$ invariant Lax operator in its factorized form (see e.g. [40])

and

$$
\mathcal{L}^{+} \equiv \mathrm{U}_{1}^{-1}\left(\begin{array}{cccc}
z_{1}-\frac{n-1}{2} & & & \\
\mathbf{D}_{2,1} & \ddots & & \\
\vdots & \ddots & \ddots & \\
\mathbf{D}_{n, 1} & \cdots & \mathbf{D}_{n, n-1} & z_{n}-\frac{n-1}{2}
\end{array}\right) \mathrm{U}_{1}
$$

$$
\mathbb{G}_{\mathcal{L}} \equiv \mathrm{U}_{n+1}
$$

\section{Appendix C. Hasse diagrams}

The quadratic functional relations (5.12) possess an interesting graphical interpretation, cf. Fig. 5. The full set of functional equations is then nicely depicted by so-called Hasse diagrams, cf. [16] and references therein. These diagrams are used to represent partially ordered sets. In our case we take a power set of $\{1, \ldots, n\}$ with order given by the inclusion relation, namely $A<B \Leftrightarrow A \subset B$. Then $\mathbf{Q}_{I}$ will inherit this ordering, giving us a partially ordered set containing all $\mathbf{Q}$-operators. By way of example, Hasse diagrams for $n=2,3,4$ are presented in the Figs. 3, 1 and 4, respectively. To read off the functional relations it is enough to take any 4-cycle in these diagrams, using the equivalence depicted in Fig. 5.

Every path in the Hasse diagram which leads from $\mathbf{Q}_{\varnothing}$ to $\mathbf{Q}_{\{1, \ldots, n\}}$ defines a system of equivalent but distinct nested Bethe equations. To find each such system, it is enough to take all Q-operators on a given path and write one relation for any three subsequent functions on the path. Such relation can be written for every three subsequent Q's because there always exists a unique 4-cycle containing them. Finally, it is interesting to point out that the Hasse diagram corresponding to the $\mathfrak{g l}(n)$ algebra forms an $n$-dimensional ordered hypercube.

\section{Appendix D. Energy formula}

Let us choose a path on the Hasse diagram given by the ordered set $\left\{a_{1}, \ldots, a_{n}\right\}=\{1, \ldots, n\}$. We define $I_{k}=I_{k-1} \cup\left\{a_{k}\right\}$ with $I_{0}=\varnothing$. The formula for the energy in terms of the Bethe roots depends only on the last-level Q's and is given by 


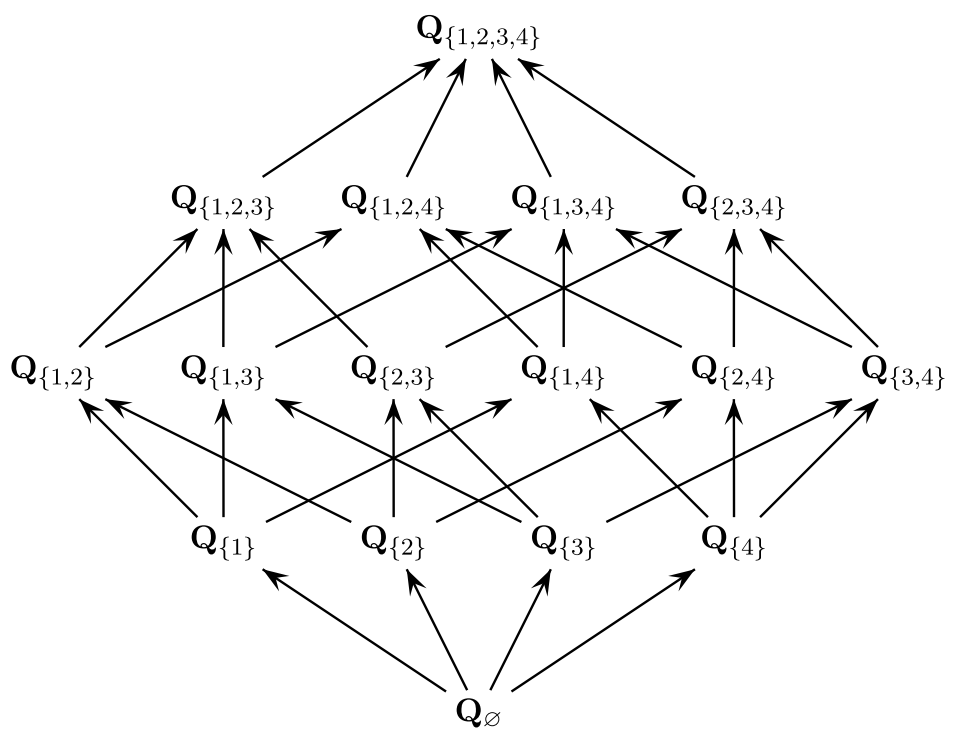

Fig. 4. Hasse diagram for $\mathfrak{g l}(4)$.

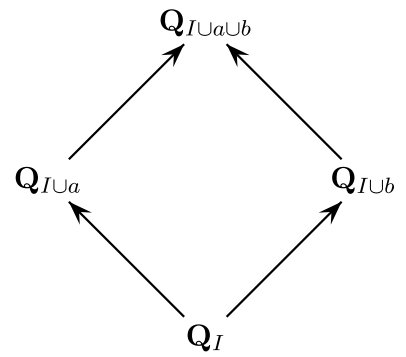

$2 i \sin \frac{\varphi_{a b}}{2} \mathbf{Q}_{I \cup a \cup b}(z) \mathbf{Q}_{I}(z)=\mathbf{Q}_{I \cup a}\left(z-\frac{1}{2}\right) \mathbf{Q}_{I \cup b}\left(z+\frac{1}{2}\right)-\mathbf{Q}_{I \cup b}\left(z-\frac{1}{2}\right) \mathbf{Q}_{I \cup a}\left(z+\frac{1}{2}\right)$

Fig. 5. Graphical depiction of the functional relations (5.12).

$$
E=2 \sum_{k=1}^{m_{I_{n-1}}} \frac{1}{\frac{1}{4}-\left(z_{k}^{I_{n-1}}\right)^{2}}
$$

where $m_{I_{n-1}}$ is the number of roots of the $\mathrm{Q}_{I_{n-1}}(z)$ function. To prove (6.8) it is enough to take the functional relation

$$
\begin{aligned}
& \frac{\mathrm{X}_{I_{k}}\left(z_{0}, z_{2}, \ldots, z_{k}\right)}{\mathrm{X}_{I_{k}}\left(z_{1}, z_{2}, \ldots, z_{k}\right)} \\
& \quad=\frac{\mathrm{X}_{I_{k}}\left(z_{0}, z_{1}, \ldots, z_{k-1}\right)}{\mathrm{X}_{I_{k}}\left(z_{1}, z_{2}, \ldots, z_{k}\right)} \frac{\mathrm{X}_{I_{k-1}}\left(z_{2}, \ldots, z_{k}\right)}{\mathrm{X}_{I_{k-1}}\left(z_{1}, z_{2}, \ldots, z_{k-1}\right)}+\frac{\mathrm{X}_{I_{k-1}}\left(z_{0}, z_{2}, \ldots, z_{k-1}\right)}{\mathrm{X}_{k-1}\left(z_{1}, z_{2}, \ldots, z_{k-1}\right)}
\end{aligned}
$$

where $\mathrm{X}_{I_{k}}$ is an eigenvalue of $\mathbf{X}_{I_{k}}$ defined in (4.11). It holds for $k=1, \ldots, n$ and originates from the fact that every $\mathrm{X}_{I_{k}}$ can be written as a determinant of partonic Q-functions as (see (5.8))

$$
\Delta_{I_{k}} \mathrm{X}_{I_{k}}\left(z_{1}, \ldots, z_{k}\right)=\operatorname{det}_{a, b \in I_{k}} \mathrm{Q}_{a}\left(z_{b}\right) .
$$


In this case the relation (D.2) is just a version of the Plücker relations and is valid for any numbers $z_{a}$. In (D.2) we omitted an explicit dependence on the spectral parameter which can be recover for every $X_{I_{k}}$ just by taking the mean value of all $z$ 's present there.

If we take $k=n$ and put $z_{a}=z-a+\frac{n+1}{2}$ then using this relation recursively we get

$$
\frac{\mathrm{T}_{\square}\left(z+\frac{1}{n}\right)}{\mathrm{Q}_{I_{n}}(z)}=\sum_{k=0}^{n-1} \frac{\mathrm{Q}_{I_{n-k}}\left(z+1+\frac{k}{2}\right)}{\mathrm{Q}_{I_{n-k}}\left(z+\frac{k}{2}\right)} \frac{\mathrm{Q}_{I_{n-k-1}}\left(z-\frac{1}{2}+\frac{k}{2}\right)}{\mathrm{Q}_{I_{n-k-1}}\left(z+\frac{1}{2}+\frac{k}{2}\right)}
$$

where $\mathrm{T}_{\square}$ denotes an eigenvalue of the transfer matrix in the fundamental representation. In order to prove (6.8) it is enough to use the well-known relation

$$
E=2 L-\left.2 \frac{d}{d z} \log \mathrm{T}_{\square}\left(z+\frac{1}{n}\right)\right|_{z=0}
$$

and the fact that $\mathrm{Q}_{I_{n}}(z)=z^{L}$ where $L$ is the length of the spin chain.

\section{References}

[1] R.J. Baxter, Partition function of the eight-vertex lattice model, Annals Phys. 70 (1972) 193-228.

[2] R.J. Baxter, Exactly Solved Models in Statistical Mechanics, Academic Press, 1982.

[3] L.D. Faddeev, How algebraic Bethe Ansatz works for integrable model, arXiv:hep-th/9605187.

[4] V.V. Bazhanov, Y.G. Stroganov, Chiral Potts model as a descendant of the six vertex model, J. Stat. Phys. 59 (1990) 799-817.

[5] M. Gaudin, V. Pasquier, The periodic Toda chain and a matrix generalization of the Bessel function's recursion relations, J. Phys. A 25 (1992) 5243-5252.

[6] V.V. Bazhanov, S.L. Lukyanov, A.B. Zamolodchikov, Integrable structure of conformal field theory II. Q-operator and DDV equation, Commun. Math. Phys. 190 (1997) 247-278, arXiv:hep-th/9604044.

[7] F.A. Smirnov, Dual Baxter equations and quantization of the affine Jacobian, J. Phys. A 33 (16) (2000) 3385-3405, http://dx.doi.org/10.1088/0305-4470/33/16/323.

[8] L.D. Faddeev, R.M. Kashaev, A.Y. Volkov, Strongly coupled quantum discrete Liouville theory. I: Algebraic approach and duality, Commun. Math. Phys. 219 (2001) 199-219, arXiv:hep-th/0006156.

[9] S.E. Derkachov, G.P. Korchemsky, A.N. Manashov, Baxter Q-operator and separation of variables for the open $S L(2, R)$ spin chain, JHEP 0310 (2003) 053, arXiv:hep-th/0309144.

[10] A.G. Bytsko, J. Teschner, Quantization of models with non-compact quantum group symmetry: Modular XXZ magnet and lattice sinh-Gordon model, J. Phys. A 39 (2006) 12927-12981, arXiv:hep-th/0602093.

[11] V.V. Bazhanov, T. Lukowski, C. Meneghelli, M. Staudacher, A shortcut to the Q-operator, J. Stat. Mech. 1011 (2010) P11002, arXiv:1005.3261 [hep-th].

[12] R. Frassek, T. Lukowski, C. Meneghelli, M. Staudacher, Oscillator construction of $s u(n \mid m)$ Q-operator, arXiv: 1012.6021 [math-ph].

[13] B. Sutherland, Model for a multicomponent quantum system, Phys. Rev. B 12 (1975) 3795-3805.

[14] C. Lai, Lattice gas with nearest-neighbor interaction in one dimension with arbitrary statistics, J. Math. Phys. 15 (1974) 1675-1676.

[15] G.P. Pronko, Y.G. Stroganov, Bethe equations “on the wrong side of equator”, J. Phys. A 32 (1999) 2333-2340, arXiv:hep-th/9808153.

[16] Z. Tsuboi, Solutions of the T-system and Baxter equations for supersymmetric spin chains, Nucl. Phys. B 826 (2010) 399-455, arXiv:0906.2039 [math-ph].

[17] I. Krichever, O. Lipan, P. Wiegmann, A. Zabrodin, Quantum integrable models and discrete classical Hirota equations, Commun. Math. Phys. 188 (1997) 267-304, arXiv:hep-th/9604080.

[18] G.P. Pronko, Y.G. Stroganov, The complex of solutions of the nested Bethe ansatz: The A(2) spin chain, arXiv: hep-th/9902085.

[19] V.V. Bazhanov, A.N. Hibberd, S.M. Khoroshkin, Integrable structure of $W(3)$ conformal field theory, quantum Boussinesq theory and boundary affine Toda theory, Nucl. Phys. B 622 (2002) 475-547, arXiv:hep-th/0105177.

[20] P. Dorey, C. Dunning, R. Tateo, Differential equations for general SU(n) Bethe ansatz systems, J. Phys. A 33 (2000) 8427-8442, arXiv:hep-th/0008039. 
[21] N. Gromov, V. Kazakov, S. Leurent, Z. Tsuboi, Wronskian solution for AdS/CFT Y-system, arXiv:1010.2720 [hep-th].

[22] V.V. Bazhanov, S.L. Lukyanov, A.B. Zamolodchikov, Integrable structure of conformal field theory. III: The YangBaxter relation, Commun. Math. Phys. 200 (1999) 297-324, hep-th/9805008.

[23] V.V. Bazhanov, Z. Tsuboi, Baxter's Q-operators for supersymmetric spin chains, Nucl. Phys. B 805 (2008) 451-516, arXiv:0805.4274 [hep-th].

[24] T. Kojima, Baxter's Q-operator for the W-algebra $W_{N}$, J. Phys. A 41 (2008) 355206, arXiv:0803.3505.

[25] V.G. Drinfel'd, Hopf algebras and the quantum Yang-Baxter equation, Doklady Akademii Nauk SSSR 283 (5) (1985) 1060-1064.

[26] A. Molev, Yangians and classical Lie algebras, Mathematical Surveys and Monographs, vol. 143, American Mathematical Society, Providence, RI, 2007.

[27] T.H. Koornwinder, V.B. Kuznetsov, Gauss hypergeometric function and quadratic $R$-matrix algebras, St. Petersburg Math. J. 6 (3) (1995) 595-618.

[28] V.B. Kuznetsov, M. Salerno, E.K. Sklyanin, Quantum Bäcklund transformation for the integrable DST model, J. Phys. A 33 (2000) 171-189, arXiv:solv-int/9908002.

[29] A.E. Kovalsky, G.P. Pronko, Baxter Q-operators for the integrable discrete self-trapping chain, Theor. Math. Phys. 142 (2008) 259-269, arXiv:nlin/0203030.

[30] V.V. Bazhanov, S.M. Khoroshkin, unpublished, 2001.

[31] H. Boos, F. Göhmann, A. Klümper, K.S. Nirov, A.V. Razumov, Exercises with the universal R-matrix, J. Phys. A Math. Gen. 43 (2010) 5208, arXiv:1004.5342 [math-ph].

[32] V.O. Tarasov, The structure of quantum $L$-operators for the $R$-matrix of the XXZ-model, Theor. Math. Phys. 61 (2) (1984) 1065-1072.

[33] V.O. Tarasov, Irreducible monodromy matrices for an $R$-matrix of the XXZ model, and lattice local quantum Hamiltonians, Theor. Math. Phys. 63 (2) (1985) 440-454.

[34] S. Derkachov, D. Karakhanyan, R. Kirschner, P. Valinevich, Iterative construction of $U_{q}(s \ell(n+1))$ representations and Lax matrix factorisation, Lett. Math. Phys. 85 (2008) 221-234, arXiv:0805.4724 [hep-th].

[35] I.N. Bernstein, I.M. Gelfand, S.I. Gelfand, Differential Operators on the Base Affine Space and the Study of gModules, Lie Groups and Their Representations, Halsted Press, 1975, pp. 21-64.

[36] V. Kazakov, A.S. Sorin, A. Zabrodin, Supersymmetric Bethe ansatz and Baxter equations from discrete Hirota dynamics, Nucl. Phys. B 790 (2008) 345-413, arXiv:hep-th/0703147.

[37] S.E. Derkachov, A.N. Manashov, Noncompact $s l(N)$ spin chains: Alternating sum representation for finite dimensional transfer matrices, arXiv:1008.4734 [nlin.SI].

[38] V. Kazakov, S. Leurent, Z. Tsuboi, Baxter's Q-operators and operatorial Backlund flow for quantum (super)-spin chains, arXiv:1010.4022 [math-ph].

[39] V. Kazakov, P. Vieira, From characters to quantum (super)spin chains via fusion, JHEP 0810 (2008) 050, arXiv: 0711.2470 [hep-th].

[40] S.E. Derkachov, A.N. Manashov, R-Matrix and Baxter Q-operators for the noncompact $S L(N, C)$ invariant spin chain, arXiv:nlin/0612003. 